\title{
Alguns resultados sobre otimização ergódica em espaços não compactos
}

\author{
Tatiane Cardoso Batista
}

DissertaÇÃo APRESEntada

$\mathrm{AO}$

Instituto de Matemática e EstatísticA

DA

Universidade de SÃo Paulo

PARA

OBTENÇÃO DO TÍTULO

$\mathrm{DE}$

Mestre em CiênCias

Programa: Matemática Aplicada

Orientador: Prof. Dr. Fabio Armando Tal

Durante o desenvolvimento deste trabalho o autor recebeu auxílio financeiro do CNPq

São Paulo, julho de 2009 



\title{
Alguns resultados sobre otimização ergódica em espaços não compactos
}

\author{
Este exemplar corresponde à redação \\ final da dissertação devidamente corrigida \\ e defendida por Tatiane Cardoso Batista \\ e aprovada pela Comissão Julgadora.
}

Banca Examinadora:

- Prof. Dr. Fabio Armando Tal (orientador) - IME-USP.

- Prof. Dr. Clodoaldo Grotta Ragazzo - IME-USP.

- Prof. Dr. Eduardo Garibaldi - UNICAMP. 



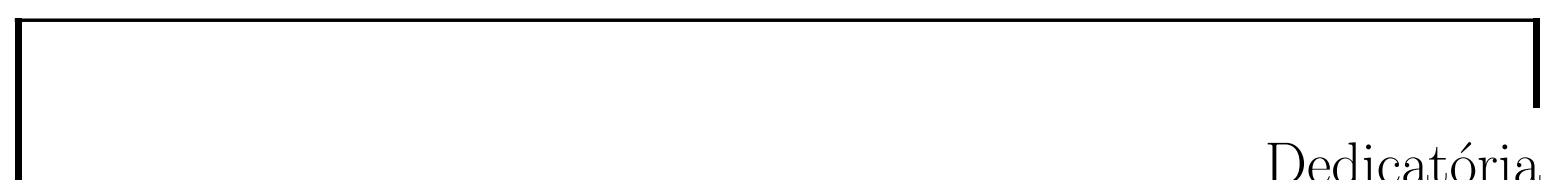

Aos meus pais,

Mauricio e Sinézia. 

Agradeço...

A Deus, sempre presente em minha vida.

Aos meus pais e meu irmão Rodolfo, por todo amor que recebi. Obrigada por acreditarem em mim e fazerem com que isso acontecesse. Amo vocês!

À toda minha família. Em especial, meus avós Benedito e Elisa, pelo carinho que recebi durante esse tempo.

Ao meu namorado Diego, que participou dessa caminhada ao meu lado.

Ao professor Dr. Fabio Tal, pelo excelente trabalho de orientação e por sua dedicação, não só durante a elaboração dessa dissertação, mas durante todo o mestrado.

Ao meu amigo Juliano, pela ajuda fundamental nesse trabalho. . . e pela paciência!

À minha amiga Débora, por me apresentar essa área e pelos agradáveis finais de semana estudando...

Aos meus amigos, Pricila, Gustavo, Rose, Gra, Humberto e Bráulio, tanto pelos momentos de estudos, quanto pela importante amizade aqui iniciada.

Aos companheiros da sala 154-B e aos amigos estrangeiros, sempre tão atenciosos.

Aos funcionários e professores do IME, pelo ambiente acolhedor proporcionado.

À Universidade de São Paulo, pela oportunidade de estudo e por toda a infraestrutura oferecida. 
Aos meus amigos UNESPIANOS, que almejaram isso junto comigo.

Ao pessoal da república, e ao meu amigo Renan, companheiros de todas as horas! Ao CNPq, pelo apoio financeiro.

A todos aqueles que contribuíram, mesmo que indiretamente, na realização desse trabalho. Muito obrigada! 


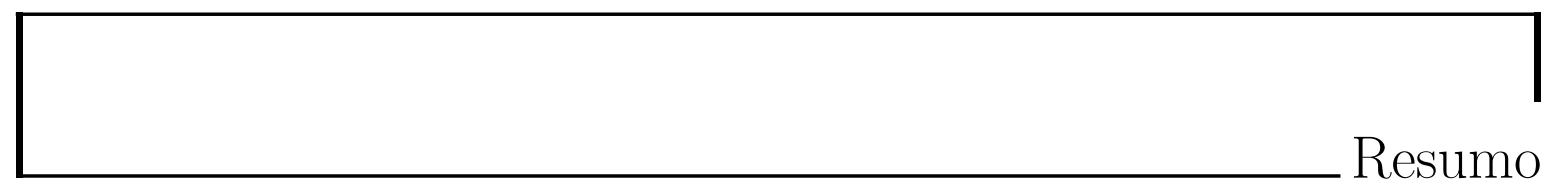

Sejam $X$ um espaço topológico não necessariamente compacto e $T: X \rightarrow X$ uma aplicação contínua. Se $f: X \rightarrow \mathbb{R}$ é contínua, daremos condições sobre $f$ que garantam a existência de medidas maximizantes caracterizadas em termos de seu suporte.

Palavras-chave: medida maximizante, forma normal, compacidade essencial. 



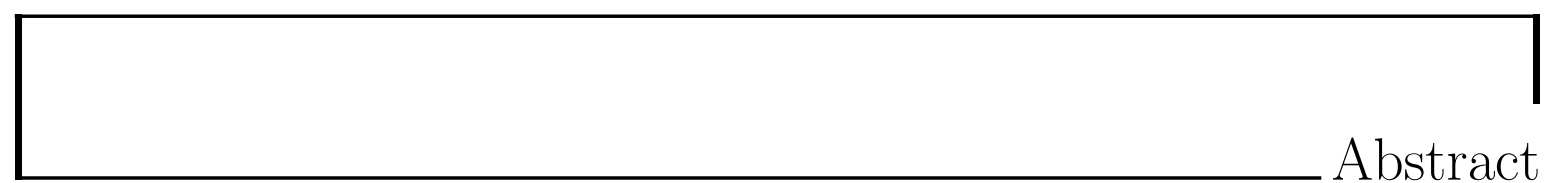

Let $X$ be a topological space not necessarily compact, and $T: X \rightarrow X$ a continuous map. If $f: X \rightarrow \mathbb{R}$ is a continuous function, we seek conditions on $f$ in order to guarantee existence of maximizing measures that are characterized in terms of its support.

Keywords: maximizing measure, normal form, essential compactness. 



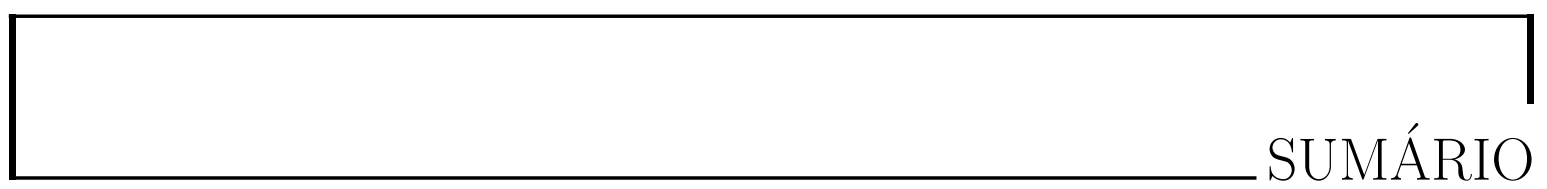

Introdução 2

1 Preliminares 4

1.1 Medidas invariantes . . . . . . . . . . . . . . . . . 4

1.2 O conjunto das medidas invariantes . . . . . . . . . . . . . . 9

1.3 Ergodicidade ........................... 14

2 Otimização ergódica 20

2.1 Motivação . . . . . . . . . . . . . . . . . . . . . 20

2.2 Média ergódica máxima e medidas maximizantes . . . . . . . . . . 26

2.3 O conjunto das medidas maximizantes . . . . . . . . . . . . . . 31

3 Otimização ergódica para sistemas dinâmicos não-compactos 34

3.1 Introdução . . . . . . . . . . . . . . . . . . . . . . . . 34

3.2 Forma normal e caracterização das medidas maximizantes . . . . . . . 40

3.3 Funções essencialmente compactas . . . . . . . . . . . . . . 51

$\begin{array}{ll}\text { Bibliografia } & 64\end{array}$ 
A teoria ergódica diz respeito a iteração de transformações $T$ que preservam medida em espaços $(X, \mathcal{B}, \mu)$ e, em particular, se o espaço é de probabilidade e $f: X \rightarrow \mathbb{R}$ é uma função integrável, estuda as médias temporais:

$$
\lim _{n \rightarrow \infty} \frac{1}{n} \sum_{i=0}^{n-1} f\left(T^{i}(x)\right) .
$$

Um dos resultados fundamentais dessa teoria é o teorema ergódico de Birkhoff, que relaciona a média temporal com a média espacial:

$$
\int_{X} f d \mu, \mu \in \mathcal{M}_{T}
$$

onde $\mathcal{M}_{T}$ é o conjunto das medidas de probabilidade $T$-invariantes.

O objeto de interesse da otimização ergódica são as medidas invariantes que maximizam a média espacial, chamadas medidas maximizantes.

Considere $T: X \rightarrow X$ contínua e $\mathcal{B}$ a $\sigma$-álgebra de Borel. Em nosso estudo, $X$ é um espaço topológico não necessariamente compacto e, por isso, são colocadas condições sobre a função $f$ que garantam a existência de medida maximizante.

O capítulo 1 trata dos conceitos básicos da teoria ergódica, estudando sistemas dinâmicos munidos de medidas invariantes. Em geral, $\mathcal{M}_{T}$ pode ser vazio, mas se $X$ é um espaço metrizável, compacto e não vazio, então $\mathcal{M}_{T} \neq \emptyset$. 
O capítulo 2 introduz noções de otimização ergódica e apresenta alguns resultados considerando a compacidade do espaço $X$. Nesse caso, o conjunto das medidas maximizantes para $f$, denotado por $\mathcal{M}_{\max }(f)$, é não vazio.

O capítulo 3 é dedicado ao estudo da otimização ergódica em espaços não necessariamente compactos. Verificamos que é suficiente $f$ ter uma forma normal para que exista medida maximizante, e que tais medidas são caracterizadas em termos de seu suporte. No caso em que $X$ é compacto e metrizável, basta que $f$ tenha uma forma do ponto fixo para garantir a existência de uma forma normal, mas no caso mais geral, onde $X$ é um espaço polonês, é necessário a compacidade essencial como condição adicional. Esses são resultados de Jenkinson, Mauldin e Urbański, provados em [6]. 


\section{CAPÍTULO 1}

Preliminares

Neste capítulo lembraremos os conceitos da Teoria Ergódica necessários para o desenvolvimento da Otimização Ergódica. Algumas das demonstrações dos resultados não serão feitas, mas podem ser encontradas em [16] e [13]. As referências para as definições da Teoria da Medida, não apresentadas aqui, são [14] e [1].

\section{$1.1 \quad$ Medidas invariantes}

Denotaremos por $(X, \mathcal{B}, \mu)$ o espaço de medida onde, $X$ é um conjunto qualquer, $\mathcal{B} \subset \mathcal{P}(X)$ é a $\sigma$-álgebra considerada e $\mu$ é a medida em $(X, \mathcal{B})$. Além disso, se $\mu(X)=1$, o espaço é chamado de probabilidade.

Definição 1.1.1. Sejam $(X, \mathcal{B}, \mu)$ um espaço de medida e $T: X \rightarrow X$ uma transformação mensurável ${ }^{1}$. Dizemos que $T$ preserva medida ou $\mu$ é T-invariante, se para todo $A \in \mathcal{B}$,

$$
\mu\left(T^{-1}(A)\right)=\mu(A) .
$$

${ }^{1} T$ é mensurável se para todo $A \in \mathcal{B}$ tem-se que $T^{-1}(A) \in \mathcal{B}$. 
No nosso caso, estamos interessados quando $T$ é um automorfismo ${ }^{2}$ num espaço $(X, \mathcal{B}, \mu)$ onde, em geral, $X$ é um espaço topológico, $\mathcal{B}$ é a $\sigma$-álgebra de Borel e $(X, \mathcal{B}, \mu)$ é de probabilidade.

Uma outra maneira de se verificar a invariância das medidas é dada a seguir:

Lema 1.1.2. Seja $X$ um espaço métrico, $\mathcal{B}$ a $\sigma$-álgebra de Borel e $\mu$ medida de probabilidade. Considere $T: X \rightarrow X$ uma transformação contínua. Se $\int_{X} f \circ T d \mu=\int_{X} f d \mu$ para toda função continua $f: X \rightarrow \mathbb{R}$ então $\mu$ é medida T-invariante.

Para o próximo lema, lembremos do seguinte resultado de Teoria da Medida encontrado em [14]:

Teorema 1.1.3. (convergência monótona) Seja $(X, \mathcal{B}, \mu)$ um espaço de medida. Considere $f_{n}: X \rightarrow[0,+\infty]$ sequência crescente de funções mensuráveis e não negativas convergindo para uma função $f: X \rightarrow[0,+\infty]$. Então,

$$
\lim _{n \rightarrow \infty} \int_{X} f_{n} d \mu=\int_{X} f d \mu
$$

Lema 1.1.4. Se $f: X \rightarrow \mathbb{R}$ é mensurável e $\mu$ é uma medida T-invariante, então $\int_{X} f d \mu=\int_{X} f \circ T d \mu$, no sentido de que se uma das integrais existe então a outra também existe, e neste caso, elas são iguais.

\section{Demonstração.}

Primeiramente, para $f=\chi_{A}$, a função característica de $A$ com $A \subset X$ qualquer:

$$
\int_{X} \chi_{A} d \mu-\int_{X} \chi_{A} \circ T d \mu=\int_{A} 1 d \mu-\int_{X} \chi_{T^{-1}(A)} d \mu=\mu(A)-\mu\left(T^{-1}(A)\right)=0 .
$$

Se $f$ é função simples o resultado é análogo, pois $f=\sum_{i=1}^{n} a_{i} \chi_{A_{i}}$, onde $A_{i} \subset X$ são distintos e $X=\bigcup_{i=1}^{n} A_{i}$.

\footnotetext{
${ }^{2} T: X \rightarrow X$ é um isomorfismo, ou seja, uma função bijetora.
} 
Se $f$ é uma função mensurável não negativa, existe uma sequência $\left(f_{n}\right)_{n \geq 1}$ de funções simples, com $0 \leq f_{1} \leq f_{2} \leq \ldots \leq f_{n} \leq \ldots \leq f$ e $\lim _{n \rightarrow \infty} f_{n}(x)=f(x), \forall x \in X$. Usando (1.1.3), analogamente,

$$
\begin{aligned}
\int_{X} f d \mu-\int_{X} f \circ T d \mu & =\int_{X} \lim _{n \rightarrow \infty} f_{n} d \mu-\int_{X} \lim _{n \rightarrow \infty} f_{n} \circ T d \mu \\
& =\lim _{n \rightarrow \infty}\left(\int_{X} f_{n} d \mu-\int_{X} f_{n} \circ T d \mu\right)=0 .
\end{aligned}
$$

Se $f$ é uma função mensurável qualquer, basta considerar $f=f^{+}-f^{-}$.

Vejamos alguns exemplos de medidas invariantes:

Exemplo 1.1.5. Suponha que $X$ é um espaço topológico e $p$ um ponto tal que $T^{n}(p)=p . \quad$ Seja $\delta_{x}$ a medida de Dirac associada a um ponto $x$ definida por: $\delta_{x}(A)=\left\{\begin{array}{l}1, \text { se } x \in A \\ 0, \text { se } x \notin A\end{array}\right.$. Então a medida

$$
\mu=\frac{1}{n} \sum_{j=0}^{n-1} \delta_{T^{j}(p)}
$$

é invariante por T. Lembramos que,

$$
\int_{X} f d \delta_{x}=f(x)
$$

Resolução 1.1.6. Seja $A \subset X$ um conjunto mensurável qualquer. Mostremos que $\mu(A)=\mu\left(T^{-1}(A)\right)$.

$$
\begin{aligned}
\frac{1}{n} \sum_{j=0}^{n-1} \delta_{T^{j}(p)}\left(T^{-1}(A)\right) & =\frac{1}{n} \#\left\{0 \leq j \leq n-1, T^{j}(p) \in T^{-1}(A)\right\} \\
& =\frac{1}{n} \#\left\{0 \leq j \leq n-1, T^{j+1}(p) \in A\right\} \\
& =\frac{1}{n} \#\left\{1 \leq j \leq n, T^{j}(p) \in A\right\}
\end{aligned}
$$


Mas, $\operatorname{como} T^{n}(p)=p$,

$$
\begin{aligned}
\frac{1}{n} \#\left\{1 \leq j \leq n, T^{j}(p) \in A\right\} & =\frac{1}{n} \#\left\{0 \leq j \leq n-1, T^{j}(p) \in A\right\} \\
& =\frac{1}{n} \sum_{j=0}^{n-1} \delta_{T^{j}(p)}(A) .
\end{aligned}
$$

No exemplo seguinte, usamos o teorema descrito em [13]:

Teorema 1.1.7. (extensão de Hahn-Kolmogorov) Seja $X$ um conjunto, $\mathcal{B}_{0}$ uma álgebra de subconjuntos de $X$ e $\mu_{0}: \mathcal{B}_{0} \rightarrow[0,1]$ uma medida. Então, se $\mathcal{B}$ é a $\sigma$-álgebra gerada por $\mathcal{B}_{0}$, existe uma única medida $\mu: \mathcal{B} \rightarrow[0,1]$ tal que $\left.\mu\right|_{\mathcal{B}_{0}}=\mu_{0}$.

Exemplo 1.1.8. Seja $X=\{1, \ldots, d\}^{\mathbb{N}}$, isto é, o conjunto de todas as sequências $x=\left(x_{n}\right)_{n=1}^{\infty}$ de inteiros em $\{1, \ldots, d\}$.

Se $w=\left(w_{1}, \ldots, w_{k}\right)$, com $w_{i} \in\{1, \ldots, d\}$ para $1 \leq i \leq k$, definimos em $X$ o cilindro de comprimento $k$ ( $k$-cilindro) pelo conjunto:

$$
[w]:=\left\{x \in X: x_{i}=w_{i} \text { para } 1 \leq i \leq k\right\}
$$

$A \sigma$-álgebra $\mathcal{B}$ considerada em $X$ é a extensão da álgebra gerada pela semi-álgebra dos cilindros de $X$.

Uma medida de probabilidade em $(X, \mathcal{B})$ é definida a partir de d números não negativos $p_{1}, \ldots, p_{d}$, com $\sum_{i=1}^{d} p_{i}=1$. A medida de um k-cilindro em $X$ é dada por:

$$
\mu([w])=p_{w_{1}} p_{w_{2}} \ldots p_{w_{k}}
$$

Consideramos neste espaço a aplicação shift $T: X \rightarrow X$ definida por $T\left(x_{1}, x_{2}, x_{3}, \ldots\right)=\left(x_{2}, x_{3}, \ldots\right)$. Essa função é contínua com respeito a métrica $d(x, y)=2^{-\min \left\{n: x_{n} \neq y_{n}\right\}}$ e preserva a medida $\mu$.

Resolução 1.1.9. Mostremos que Té contínua. 
Dado $\epsilon>0$, escolha $n$ tal que $2^{-(n+1)}<\epsilon$.

Tome $\delta=\frac{1}{2^{n+2}}$. Se $d(x, y)<\delta$, então $x_{j}=y_{j}, j=1, \ldots, n+1$. Assim,

$$
d(T(x), T(y))=d\left(\left(x_{2}, x_{3}, \ldots, x_{n}, \ldots\right),\left(y_{2}, y_{3}, \ldots, y_{n}, \ldots\right)\right) \leq \frac{1}{2^{n+1}}<\epsilon .
$$

Para mostrar que $T$ preserva medida, basta olhar para os cilindros:

$$
\begin{aligned}
\mu\left(T^{-1}([w])\right) & =\mu\left(\bigcup_{x_{1}=1}^{d}\left(x_{1}, w_{1}, \ldots, w_{k}, x_{k+2}, x_{k+3}, \ldots\right)\right) \\
& =\sum_{i=1}^{d} p_{i} p_{w_{1}} \ldots p_{w_{k}}=1 \cdot\left(p_{w_{1}} \ldots p_{w_{k}}\right)=\mu([w]) .
\end{aligned}
$$

O próximo teorema enunciado merece destaque por suas várias aplicações.

Teorema 1.1.10. (recorrência de Poincaré) Seja $(X, \mathcal{B}, \mu)$ espaço de probabilidade e $T$ um automorfismo que preserva $\mu$ nesse espaço. Considere $E \in \mathcal{B}$ e $\hat{E} \in \mathcal{B}$ subconjunto de $E$ tal que seus pontos retornam infinitas vezes a $E$, isto é,

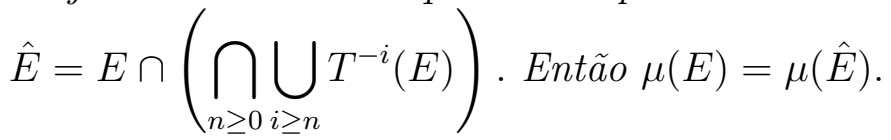

Demonstração. Considere $E_{n}=\bigcup_{i \geq n} T^{-i}(E)$, para $n \geq 0$.

Observe que $E_{0} \supset E_{1} \supset \ldots \supset E_{n} \supset \ldots$

Além disso, $T^{-1}\left(E_{i}\right)=E_{i+1}$, ou seja,

$$
\mu\left(E_{i}\right)=\mu\left(T^{-1}\left(E_{i}\right)\right)=\mu\left(E_{i+1}\right) .
$$

Segue que

$$
\mu\left(\bigcap_{n \geq 0} E_{n}\right)=\lim _{N \rightarrow \infty} \mu\left(E_{N}\right)=\lim _{N \rightarrow \infty} \mu\left(E_{0}\right)=\mu\left(E_{0}\right) .
$$

Como $\bigcap_{n \geq 0} E_{n} \subset E_{0}$ e vale a igualdade acima, então existe $B \in \mathcal{B}, \operatorname{com} \mu(B)=0$, tal que:

$$
E_{0}=\bigcap_{n \geq 0} E_{n} \cup B
$$




$$
\Rightarrow \bigcap_{n \geq 0} E_{n}=E_{0} \cap B^{C}
$$

$\operatorname{com} \mu\left(B^{C}\right)=1$. Portanto,

$$
\mu(\hat{E})=\mu\left(E \cap \bigcap_{n \geq 0} E_{n}\right)=\mu\left(E \cap E_{0} \cap B^{C}\right) \stackrel{E \subseteq E_{0}}{=} \mu\left(E \cap B^{C}\right)=\mu(E) .
$$

A versão métrica desse resultado diz que, se $X$ é um espaço métrico separável e $\mathcal{B}$ a $\sigma$-álgebra de Borel, então $\mu$-quase todo ponto é recorrente, ou seja,

$$
\mu(\{p \in X: p \notin \omega(p)\})=0,
$$

onde $\omega(p)$ é chamado de $\omega$-limite ${ }^{3}$ de $p$.

\section{$1.2 \mathrm{O}$ conjunto das medidas invariantes}

Considere $X$ um espaço métrico compacto e $\mathcal{M}(X)$ o conjunto de todas as medidas de probabilidade sobre os borelianos de $X$. Para uma transformação $T: X \rightarrow X$, definimos $\mathcal{M}_{T}(X) \subset \mathcal{M}(X)$ o conjunto das medidas invariantes por $T$.

Para estudar $\mathcal{M}(X)$, será útil introduzir uma métrica nesse conjunto. Para isso, lembremos dos seguintes teoremas encontrados respectivamente em [14] e [10]:

Teorema 1.2.1. (representação de Riesz) Se X é espaço métrico compacto e se $\xi: \mathcal{C}^{0}(X) \rightarrow \mathbb{R}$ é funcional linear, contínuo, positivo, com $\xi(1)=1$, então existe uma única probabilidade $\mu \in \mathcal{M}(X)$ tal que

$$
\int_{X} f d \mu=\xi(f)
$$

para toda $f \in \mathcal{C}^{0}(X)$.

\footnotetext{
${ }^{3}$ Lembremos que $\omega(p):=\left\{x \in X: \exists\left\{n_{k}\right\}_{k \in \mathbb{N}} \subset \mathbb{N}\right.$ tal que $n_{k} \rightarrow \infty$ e $\left.\lim _{k \rightarrow \infty} f^{n_{k}}(x)=p\right\}$
} 
Teorema 1.2.2. Seja E um espaço normado separável e seja $E^{*}$ o espaço dual ${ }^{4}$ a . Considere $S$ a bola unitária fechada de $E^{*}$.

A topologia induzida em $S$ pela topologia fraca- ${ }^{*}$ pode ser definida mediante a métrica

$$
d\left(\xi_{1}, \xi_{2}\right)=\sum_{n=1}^{\infty} 2^{-n}\left|\left(\xi_{1}-\xi_{2}\right)\left(z_{n}\right)\right|,
$$

onde $\left\{z_{n}\right\}$ é um conjunto enumerável e denso na bola unitária de E.

Assim, consideremos $\mathcal{C}^{0}(X)$ o espaço vetorial das funções contínuas provido da norma do supremo $\|f\|_{\infty}=\sup _{x \in X}|f(x)|$. Como $X$ é métrico compacto, existe um conjunto enumerável $\left\{g_{i}\right\}_{i \in \mathbb{N}}$ denso na bola unitária

$$
B=\left\{f \in \mathcal{C}^{0}(X):\|f\|_{\infty} \leq 1\right\}
$$

Consideremos também o espaço dual de $\mathcal{C}^{0}(X)$. Dado $\xi \in \mathcal{C}^{0}(X)^{*}$ nas condições de 1.2.1, existe uma única medida de probabilidade $\mu \in \mathcal{M}(X)$ tal que

$$
\int_{X} f d \mu=\xi(f)
$$

para toda $f \in \mathcal{C}^{0}(X)$. Ou seja, $\mathcal{M}(X) \subseteq \mathcal{C}^{0}(X)^{*}$.

Tomando o conjunto $\left\{g_{i}\right\}_{i \in \mathbb{N}}$, teremos que $\|\xi\|_{\infty}=\sup _{\left\|g_{i}\right\|=1}\left|\xi\left(g_{i}\right)\right| \leq 1$, e utilizando 1.2.2, definimos em $\mathcal{M}(X)$ a seguinte métrica:

$$
d(\mu, v)=\sum_{i=1}^{\infty} \frac{1}{2^{i}}\left|\int_{X} g_{i} d \mu-\int_{X} g_{i} d v\right| .
$$

Para mostrar a convergência em $\mathcal{M}(X)$ podemos usar o seguinte lema:

Lema 1.2.3. Seja $X$ espaço métrico compacto e $\left\{g_{i}\right\}_{i \in \mathbb{N}}$ um conjunto enumerável denso na bola unitária $B . S e\left\{\mu_{n}\right\}_{n} \subset \mathcal{M}(X)$ é sequência de probabilidades, então são equivalentes:

\footnotetext{
${ }^{4} \mathrm{O}$ espaço dual $E^{*}$ é constituído de todos os funcionais lineares contínuos definidos num espaço normado $E$.
} 
1. $\lim _{n \rightarrow \infty} d\left(\mu_{n}, \mu\right)=0$

2. $\lim _{n \rightarrow \infty} \int_{X} g_{i} d \mu_{n}=\int_{X} g_{i} d \mu$, para todo $i \geq 1$.

3. $\lim _{n \rightarrow \infty} \int_{X} g d \mu_{n}=\int_{X} g d \mu$, para toda $g \in \mathcal{C}^{0}(X)$.

Outro resultado muito importante que pode ser visto em [10] é:

Teorema 1.2.4. (Banach-Alaoglu) Seja E um espaço normado separável e seja $E^{*}$ o espaço dual a E. Toda bola fechada de $E^{*}$ é compacta na topologia fraca-* .

A consequência direta desse teorema é,

Teorema 1.2.5. $\mathcal{M}(X)$ é um espaço métrico compacto (com a métrica definida anteriormente).

Demonstração. Basta mostrar que $\mathcal{M}(X)$ é fechado em $\mathcal{C}^{0}(X)^{*}$.

Considere $\mu_{n} \in \mathcal{M}(X) \operatorname{com} \mu_{n} \rightarrow \mu$.

Por 1.2.3, $\lim _{n \rightarrow \infty} \int_{X} g d \mu_{n}=\int_{X} g d \mu$, para toda $g \in \mathcal{C}^{0}(X)$. Em particular,

$$
\lim _{n \rightarrow \infty} \mu_{n}(X)=\mu(X)
$$

Logo, $\mu$ é uma medida de probabilidade, ou seja, $\mu \in \mathcal{M}(X)$.

Sobre o conjunto das medidas invariantes, podemos concluir:

Teorema 1.2.6. (Krylov-Bogolioubov) $\mathcal{M}_{T}(X) \neq \emptyset$.

\section{Demonstração.}

Consideremos a sequência $\left\{\mu_{n}\right\} \subset \mathcal{M}(X)$ dada por:

$$
\mu_{n}=\frac{1}{n} \sum_{k=0}^{n-1} \delta_{T^{k} x}
$$


Por causa da compacidade de $\mathcal{M}(X)$, existe subsequência $\left\{\mu_{n_{m}}\right\}$ que converge na topologia fraca-* a uma medida de probabilidade $\mu$, isto é,

$$
\int_{X} g d \mu=\lim _{m \rightarrow \infty} \int_{X} g d \mu_{n_{m}}
$$

para qualquer função contínua g.

Para verificar que $\mu$ é uma medida invariante, é suficiente mostrar que $\int_{X} g \circ T d \mu=\int_{X} g d \mu$ para toda função contínua $g$ (1.1.2). Temos que $T$ é contínua então $g \circ T$ também é, e então,

$$
\begin{aligned}
\int_{X} g \circ T d \mu & =\lim _{m \rightarrow \infty} \int_{X} g \circ T d \mu_{n_{m}} \\
& =\lim _{m \rightarrow \infty} \frac{1}{n_{m}} \sum_{k=0}^{n_{m}-1} g\left(T^{k+1}(x)\right) \\
& =\lim _{m \rightarrow \infty}\left(\frac{1}{n_{m}} \sum_{k=0}^{n_{m}-1} g\left(T^{k}(x)\right)+\frac{1}{n_{m}}\left[g\left(T^{n_{m}}(x)\right)-g(x)\right]\right) .
\end{aligned}
$$

Como $g$ é limitada em $X$,

$$
\int_{X} g \circ T d \mu=\lim _{m \rightarrow \infty} \frac{1}{n_{m}} \sum_{k=0}^{n_{m}-1} g\left(T^{k}(x)\right)=\lim _{m \rightarrow \infty} \int_{X} g d \mu_{n_{m}}=\int_{X} g d \mu .
$$

Logo, $\mathcal{M}_{T}(X) \neq \emptyset$.

Lema 1.2.7. $\mathcal{M}_{T}(X)$ é convexo e compacto.

\section{Demonstração.}

Mostremos que $\mathcal{M}_{T}(X)$ é convexo, ou seja, se para quaisquer $\mu_{1}, \mu_{2} \in \mathcal{M}_{T}(X)$ e para qualquer $t \in[0,1]$,

$$
\mu_{t}=(1-t) \mu_{1}+t \mu_{2} \in \mathcal{M}_{T}(X)
$$

De fato,

$$
\mu_{t}(A)=(1-t) \mu_{1}(A)+t \mu_{2}(A)=(1-t) \mu_{1}\left(T^{-1} A\right)+t \mu_{2}\left(T^{-1} A\right)=\mu_{t}\left(T^{-1} A\right)
$$


para todo $A \subset X$ mensurável.

Mostremos que $\mathcal{M}_{T}(X)$ é fechado, ou seja, se $\mu_{n} \in \mathcal{M}_{T}(X)$ com $\mu_{n} \rightarrow \mu$, então $\mu \in \mathcal{M}_{T}(X)$.

De fato, considerando $f$ uma função contínua qualquer e usando 1.2.3,

$$
\int_{X} f \circ T d \mu=\lim _{n \rightarrow \infty} \int_{X} f \circ T d \mu_{n}=\lim _{n \rightarrow \infty} \int_{X} f d \mu_{n}=\int_{X} f d \mu
$$

Logo, por 1.1.2, $\mu$ é uma medida invariante.

Além disso, temos que todo subconjunto fechado de um espaço topológico compacto é compacto [14]. Como $\mathcal{M}(X)$ é compacto, então $\mathcal{M}_{T}(X) \subset \mathcal{M}(X)$ é compacto.

O teorema seguinte será utilizado posteriormente no trabalho.

Teorema 1.2.8. Seja $(X, \mathcal{B}, \mu)$ um espaço de probabilidade, onde $X$ é um espaço métrico compacto e $\mathcal{B}$ é a $\sigma$-álgebra de Borel. Se $\mu_{n} \rightarrow \mu$ na topologia fraca-*, então, para todo $F \in \mathcal{B}$ fechado,

$$
\limsup _{n \rightarrow \infty} \mu_{n}(F) \leq \mu(F) .
$$

Demonstração. Defina os abertos:

$$
U_{k}=\left\{x \in X: d(x, F)<\frac{1}{k}\right\}
$$

para todo $k \geq 1$

Temos que toda medida finita é contínua [15], ou seja, é contínua por cima. Logo, medidas de probabilidades são contínuas por cima ${ }^{5}$ :

$$
\lim _{k \rightarrow \infty} \mu\left(U_{k}\right)=\mu(F)
$$

\footnotetext{
${ }^{5}$ Uma medida $\mu: \mathcal{B} \rightarrow[0, \infty]$ é contínua por cima se para toda sequência $\left\{E_{i}\right\} \subset \mathcal{B}, \operatorname{com} E_{i+1} \subset E_{i}$, tal que $\bigcap_{i=1}^{\infty} E_{i}=E$, vale $\lim _{i \rightarrow \infty} \mu\left(E_{i}\right)=\mu(E)$. Ela é contínua por baixo se para toda sequência $\left\{E_{i}\right\} \subset \mathcal{B}$, com $E_{i} \subset E_{i+1}$, tal que $\bigcup_{i=1}^{\infty} E_{i}=E$, vale $\lim _{i \rightarrow \infty} \mu\left(E_{i}\right)=\mu(E)$.
} 
onde $F=\bigcap_{k=1}^{\infty} U_{k}$.

Tome $f_{k} \in \mathcal{C}^{0}(X)$ tal que $0 \leq f_{k} \leq 1$ em $U_{k} \backslash F, f_{k}=1$ em $F$ e $f_{k}=0$ em $X \backslash U_{k}$. Então,

$$
\begin{aligned}
\limsup _{n \rightarrow \infty} \mu_{n}(F) & =\limsup _{n \rightarrow \infty} \int_{X} \chi_{F} d \mu_{n} \leq \limsup _{n \rightarrow \infty} \int_{X} f_{k} d \mu_{n} \stackrel{1.2 .3}{=} \int_{X} f_{k} d \mu \\
& =\int_{X \backslash U_{k}} f_{k} d \mu+\int_{U_{k} \backslash F} f_{k} d \mu+\int_{F} f_{k} d \mu \leq \mu\left(U_{k}\right)
\end{aligned}
$$

Em particular, quando $k \rightarrow \infty$,

$$
\limsup _{n \rightarrow \infty} \mu_{n}(F) \leq \mu(F)
$$

\subsection{Ergodicidade}

Um resultado fundamental da teoria ergódica é o teorema que diz respeito a média temporal:

Teorema 1.3.1. (ergódico de Birkhoff) Seja $(X, \mathcal{B}, \mu)$ um espaço de probabilidade e $T: X \rightarrow X$ uma transformação que preserva medida. Então,

1. se $f \in \mathcal{L}^{1}(X)$, o limite

$$
\tilde{f}(x):=\lim _{n \rightarrow \infty} \frac{1}{n} \sum_{j=0}^{n-1} f\left(T^{j}(x)\right)
$$

existe para quase todo ponto $x \in X$.

2. $\tilde{f}$ é uma função invariante, ou seja, $\tilde{f}(x)=\tilde{f}(T(x))$ quase todo ponto.

3. $\int_{X} \tilde{f} d \mu=\int_{X} f d \mu$.

4. $\lim _{n \rightarrow \infty}\left\|\tilde{f}-\frac{1}{n} \sum_{j=0}^{n-1} f \circ T^{j}\right\|_{1}=0$ 
A seguir, veremos que a ergodicidade é condição suficiente e necessária para essa média temporal ser igual a média espacial em quase todo ponto. Mais precisamente, se $T$ é ergódica e $f \in \mathcal{L}^{1}(X)$,

$$
\lim _{n \rightarrow \infty} \frac{1}{n} \sum_{i=0}^{n-1} f\left(T^{i}(x)\right)=\int_{X} f d \mu,
$$

em $\mu$-quase todo ponto $x \in X$.

Definição 1.3.2. Considere $T$ um automorfismo que preserva medida em $(X, \mathcal{B}, \mu)$.Um conjunto $A \in \mathcal{B}$ é dito invariante se $T^{-1}(A)=A$.

Definição 1.3.3. Seja $(X, \mathcal{B}, \mu)$ um espaço de probabilidade. Uma transformação $T$ que preserva medida nesse espaço é chamada ergódica se todo conjunto invariante tem medida zero ou um.

Lema 1.3.4. Seja $T$ uma transformação que preserva medida em um espaço de probabilidade $(X, \mathcal{B}, \mu)$. T é ergódica se, e somente se, qualquer função mensurável $f: X \rightarrow \mathbb{R}$ invariante $\mu$-q.t.p. for constante $\mu$-q.t.p.

Demonstração. Primeiramente, suponha que toda função mensurável $f: X \rightarrow \mathbb{R}$ invariante $\mu$-q.t.p. é constante $\mu$-q.t.p.

Considere $B \in \mathcal{B}$ um conjunto invariante e tome $f=\chi_{B}$. Vejamos que $f$ é invariante:

$$
\begin{aligned}
\chi_{B}(x) & =\left\{\begin{array}{l}
1, \text { se } x \in B=T^{-1}(B) \\
0, \text { se } x \notin B=T^{-1}(B)
\end{array}\right. \\
& =\left\{\begin{array}{l}
1, \text { se } T(x) \in B \\
0, \text { se } T(x) \notin B
\end{array}\right. \\
& =\chi_{B}(T(x)) .
\end{aligned}
$$

Mas, para $\chi_{B}$ ser constante $\mu$-q.t.p., $\mu(B)=0$ ou $\mu(B)=1$. Portanto, $T$ é ergódica. 
Suponha agora que existe $f: X \rightarrow \mathbb{R}$ mensurável, tal que $f=f \circ T$ quase todo ponto, e $f$ não é constante $\mu$-q.t.p. Logo, existe $M \in \mathbb{R}$ tal que, se

$$
E:=\{x \in X: f(x)<M\},
$$

então $0<\mu(E)<1$ e $0<\mu\left(E^{C}\right)<1$.

Observe que $E$ é invariante, ou seja, $T(E)=E$. De fato, se $x \in E$ então $f(T(x))=f(x)<M$, implicando que $T(x) \in E$. Analogamente, se $T(x) \in E$ então $x \in E$.

Mas, $\mu(E)$ é diferente de zero e de um. Portanto, $\mu$ não é ergódica.

O próximo lema mostra uma maneira alternativa de verificar a condição de ergodicidade.

Lema 1.3.5. Seja $T$ um automorfismo num espaço $(X, \mathcal{B}, \mu)$ que preserva medida. Então $T$ é ergódica se, e somente se, para todo $A, B \in \mathcal{B}$, vale

$$
\lim _{n \rightarrow \infty} \frac{1}{n} \sum_{i=0}^{n-1} \mu\left(T^{-i}(A) \cap B\right)=\mu(A) \mu(B) .
$$

Vejamos alguns exemplos de ergodicidade:

Exemplo 1.3.6. A medida definida em 1.1.5 é ergódica.

Resolução 1.3.7. Seja $N=\left\{p, T(p), T^{2}(p), \ldots, T^{n-1}(p)\right\}$. Observemos que $\mu(N)=1$. Tomemos $A \subset X$ um conjunto mensurável invariante.

Se $p \in A$ então $N$ está contido em $A$, e nesse caso, $\mu(A)=1$.

Se $p \notin A$ então $A$ não intersecta $N$, e nesse caso, $\mu(A)=0$.

Logo, todo conjunto invariante tem medida zero ou um, ou seja, T é ergódica.

$O$ conjunto $N$ é dito o suporte da medida $\mu$, como veremos em breve. 
Exemplo 1.3.8. Uma rotação do círculo é ergódica com respeito a medida de Lebesgue se, e somente se, for irracional.

O teorema seguinte auxiliará na caracterização das medidas de probabilidade ergódicas de $T$.

Teorema 1.3.9. Seja $X$ espaço métrico compacto e $T: X \rightarrow X$ contínua. Se $\mu$ é ergódica e $v \in \mathcal{M}_{T}(X)$ é absolutamente contínua $a^{6}$ com respeito a $\mu$ então, $v=\mu$.

Lema 1.3.10. Seja $(X, \mathcal{B}, \mu)$ um espaço de medida e $T: X \rightarrow X$ contínua, onde $X$ é um espaço métrico compacto. Então $\mu$ é ergódica se, e somente se, $\mu$ é ponto extremal de $\mathcal{M}_{T}(X) .^{7}$

Se $\mathcal{M}_{T}(X)$ consistir de um único elemento, $T$ é dita unicamente ergódica.

Demonstração. Suponha que $\mu$ é ergódica e $\mu=t \mu_{1}+(1-t) \mu_{2}$, com $0<t<1$ e $\mu_{1}, \mu_{2} \in \mathcal{M}_{T}(X)$. Então,

como $\mu_{1}$ e $\mu_{2}$ são absolutamente contínuas com respeito a $\mu$, do teorema anterior segue que $\mu_{1}=\mu$ e $\mu_{2}=\mu$. Portanto, $\mu$ é ponto extremal de $\mathcal{M}_{T}(X)$.

Suponha agora que $\mu$ não é ergódica. Então existe $A \in \mathcal{B}$ um conjunto invariante com $0<\mu(A)<1$. Observemos que para todo $B \in \mathcal{B}$,

$$
\begin{aligned}
& \mu(B)=\mu(A) \cdot \frac{\mu(B \cap A)}{\mu(A)}+\mu\left(A^{C}\right) \cdot \frac{\mu\left(B \cap A^{C}\right)}{\mu\left(A^{C}\right)} \\
& \mu(B)=\mu(A) \cdot \frac{\mu(B \cap A)}{\mu(A)}+(1-\mu(A)) \cdot \frac{\mu\left(B \cap A^{C}\right)}{\mu\left(A^{C}\right)} .
\end{aligned}
$$

Assim, basta tomar $\mu_{1}(B)=\frac{\mu(B \cap A)}{\mu(A)}$ e $\mu_{2}(B)=\frac{\mu\left(B \cap A^{C}\right)}{\mu\left(A^{C}\right)}, \forall B \in \mathcal{B}$.

${ }^{6}$ A medida $v$ é absolutamente contínua com respeito a $\mu$ se $v(A)=0$ sempre que $\mu(A)=0$, para $A$ mensurável.

${ }^{7} \mu$ é ponto extremal de $\mathcal{M}_{T}(X)$ se, para quaisquer $\mu_{1}, \mu_{2} \in \mathcal{M}_{T}(X)$ e $t \in[0,1]$, a identidade $\mu=t \mu_{1}+(1-t) \mu_{2}$ implicar que $t=0$ ou $t=1$. 
Temos que $\mu_{1} \in \mathcal{M}_{T}(X)$ pois, usando que $T^{-1}(A)=A$,

$$
\mu_{1}\left(T^{-1}(B)\right)=\frac{\mu\left(T^{-1}(B) \cap A\right)}{\mu(A)}=\frac{\mu\left(T^{-1}(B \cap A)\right)}{\mu(A)}=\frac{\mu(B \cap A)}{\mu(A)}=\mu_{1}(B) .
$$

Analogamente $\mu_{2} \in \mathcal{M}_{T}(X)$.

Portanto, $\mu$ não é ponto extremal de $\mathcal{M}_{T}(X)$.

Abaixo, definiremos o suporte de uma medida, conceito essencial no nosso trabalho.

Definição 1.3.11. Sejam $X$ espaço métrico separável e $\mu$ medida de probabilidade sobre os borelianos. O suporte de $\mu$ é dado por

$$
\operatorname{supp}(\mu)=\{x \in X: \forall \text { aberto } U \operatorname{com} x \in U, \mu(U)>0\}
$$

Lema 1.3.12. O suporte de $\mu$ é um conjunto fechado e $\mu(\operatorname{supp}(\mu))=1$.

A fim de entender melhor o conjunto das medidas invariantes, considere $X$ um espaço métrico compacto e $T: X \rightarrow X$ uma transformação contínua. O teorema descrito abaixo diz que cada medida de $\mathcal{M}_{T}(X)$ pode ser escrita em termos dos pontos extremais de $\mathcal{M}_{T}(X)$, ou seja, das medidas ergódicas.

Teorema 1.3.13. (decomposição ergódica) Sejam $X$ um espaço compacto e $T: X \rightarrow X$ uma função contínua. Então, para cada $\mu \in \mathcal{M}_{T}(X)$, existe $\rho_{\mu}$ uma medida definida sobre os borelianos do espaço métrico compacto $\mathcal{M}_{T}(X)$, com a propriedade que $\rho_{\mu}\left(\operatorname{EM}_{T}(X)\right)=1$ e

$$
\mu=\int_{E \mathcal{M}_{T}(X)} m d \rho_{\mu}(m)
$$

onde $\operatorname{EM}_{T}(X)$ são as medidas ergódicas em $\mathcal{M}_{T}(X)$.

A seguir, veremos um resultado extra, importante na otimização ergódica. 
Definição 1.3.14. Uma sequência $x_{n}, n \geq 1$, é chamada subaditiva se satisfaz: $x_{n+m} \leq x_{n}+x_{m}$.

Lema 1.3.15. Para toda sequência subaditiva $x_{n}, n \geq 1$, o limite $\lim _{n \rightarrow \infty} \frac{x_{n}}{n}$ existe e é igual $a \inf _{n} \frac{x_{n}}{n}$.

\section{Demonstração.}

Sejam $m \in \mathbb{N}$ fixado e $n>m$ com $n=k m+p, 0 \leq p \leq m-1$. Então,

$$
\begin{aligned}
x_{n} & =x_{k m+p} \leq x_{k m}+x_{p}=x_{(m+\ldots+m)}+x_{p} \leq k \cdot x_{m}+x_{p} \\
\Rightarrow \quad \frac{x_{n}}{n} & \leq \frac{k \cdot x_{m}}{n}+\frac{x_{p}}{n}=\frac{k \cdot x_{m}}{k m+p}+\frac{x_{p}}{n}=\frac{x_{m}}{m+\frac{p}{k}}+\frac{x_{p}}{n} .
\end{aligned}
$$

Em particular, quando $n \rightarrow \infty, k \rightarrow \infty$ e

$$
\begin{aligned}
\limsup _{n \rightarrow \infty} \frac{x_{n}}{n} \leq \frac{x_{m}}{m} \\
\Rightarrow \limsup _{n \rightarrow \infty} \frac{x_{n}}{n} \leq \inf _{m \in N} \frac{x_{m}}{m} \leq \liminf _{n \rightarrow \infty} \frac{x_{n}}{n} .
\end{aligned}
$$

Portanto, existe o limite de $\frac{x_{n}}{n}$ e ele é igual a $\inf _{n} \frac{x_{n}}{n}$. 


\section{CAPÍTULO 2}

Otimização ergódica

Esse capítulo tratará da teoria básica utilizada no foco principal do nosso trabalho, o capítulo 3. As referências para o estudo dos resultados aqui colocados são encontradas em [6] e [8].

\subsection{Motivação}

Suponha uma aplicação $T: X \rightarrow X$ num conjunto $X$ qualquer, e uma função $f: X \rightarrow \mathbb{R}$. Seja $S_{n} f=\sum_{i=0}^{n-1} f \circ T^{i}$. Nosso interesse será determinar o valor máximo da média temporal

$$
\lim _{n \rightarrow \infty} \frac{1}{n} S_{n} f(x)
$$

Em outras palavras, estamos interessados no supremo do limite 2.1 sobre todas as escolhas da posição inicial $x$. Mais do que isso, estamos particularmente interessados em verificar se para algum $x$, o supremo é atingido.

Os pontos $x$ cujo os quais o limite em 2.1 atingem o supremo, bem como suas órbitas $\left\{x, T(x), T^{2}(x), \ldots\right\}$, serão chamados de maximizantes.

Em geral, o limite acima não precisa existir. Para contornar esse problema podemos usar dois caminhos. O primeiro é definirmos 


$$
\operatorname{Reg}(X, T, f):=\left\{x \in X: \lim _{n \rightarrow \infty} \frac{1}{n} S_{n} f(x) \text { existe }{ }^{1}\right\}
$$

e então calcularmos o supremo sobre esses pontos.

O segundo caminho é usar o lim sup no lugar do limite e, então, calcular o supremo sobre todos os pontos $x \in X$.

Exemplo 2.1.1. Tome um subconjunto $A \subset X$, e seja $f=\chi_{A}$ a função característica associada. Nesse caso, a média temporal é dada por

$$
\lim _{n \rightarrow \infty} \frac{1}{n} \#\left\{1 \leq i \leq n: T^{i}(x) \in A\right\}
$$

Se o limite existe, ele é a frequência de visitação, ou seja, a frequência com que a órbita de $x$ "fica" no conjunto $A$.

Então,

$$
\begin{gathered}
\sup _{x \in \operatorname{Reg}(X, T, f)} \lim _{n \rightarrow \infty} \frac{1}{n} \#\left\{1 \leq i \leq n: T^{i}(x) \in A\right\} \quad \text { e } \\
\sup _{x \in X} \limsup _{n \rightarrow \infty} \frac{1}{n} \#\left\{1 \leq i \leq n: T^{i}(x) \in A\right\}
\end{gathered}
$$

podem ser interpretados como a máxima frequência de visitação para o conjunto $A$. Note que os valores não precisam coincidir.

Será conveniente relacionarmos a média temporal com a média espacial. Para isso, assumiremos que $X$ é um espaço topológico, e que $T: X \quad X \quad X$ e $f: X \rightarrow \mathbb{R}$ são contínuas. Considere também:

$$
\mathcal{M}_{f}:=\left\{\mu \in \mathcal{M}_{T}: \int_{X} f d \mu \text { existe }^{2}\right\} .
$$

A partir dessas motivações, podemos definir,

\footnotetext{
${ }^{1} \mathrm{O}$ limite existe se é diferente de $+\infty$ ou $-\infty$.

${ }^{2}$ Observemos que, para alguma medida $\mu$, a integral $\int_{X} f d \mu \in[-\infty, \infty]$ existe se $\int_{X} f^{+} d \mu$ e $\int_{X} f^{-} d \mu$ são não ambas infinito.
} 
Definição 2.1.2. Seja $X$ um espaço topológico, e suponha que $f: X \rightarrow \mathbb{R}$ é contínua. Então:

$$
\begin{aligned}
& \alpha(f)=\alpha(f, T)=\sup _{m \in \mathcal{M}_{f}} \int_{X} f d m \\
& \beta(f)=\beta(f, T)=\sup _{x \in \operatorname{Reg}(X, T, f)} \lim _{n \rightarrow \infty} \frac{1}{n} S_{n} f(x) \\
& \gamma(f)=\gamma(f, T)=\sup _{x \in X} \limsup _{n \rightarrow \infty} \frac{1}{n} S_{n} f(x) \\
& \delta(f)=\delta(f, T)=\limsup _{n \rightarrow \infty} \frac{1}{n} \sup _{x \in X} S_{n} f(x) .
\end{aligned}
$$

Por convenção, o supremo do conjunto vazio será $-\infty$.

A seguir, faremos algumas observações que podem ser úteis nos estudos.

Observação 2.1.3. Se $f$ é mensurável e é limitada superiormente ou inferiormente, então $\mathcal{M}_{f}=\mathcal{M}_{T}$.

\section{Demonstração.}

Por definição, $\mathcal{M}_{f} \subset \mathcal{M}_{T}$. Provemos que $\mathcal{M}_{T} \subset \mathcal{M}_{f}$ :

Sejam $\mu \in \mathcal{M}_{T}$ e $c$ o limite superior de $f$ :

$$
\begin{aligned}
\int_{X} f^{+} d \mu \leq \int_{X} c d \mu=c \mu(X)=c<+\infty & \Rightarrow \int_{X} f d \mu \text { existe } \\
& \Rightarrow \mu \in \mathcal{M}_{f} .
\end{aligned}
$$

Analogamente se mostra que $\mathcal{M}_{T} \subset \mathcal{M}_{f}$ quando $f$ é limitada inferiormente.

Observação 2.1.4. Se $X$ é compacto, então $\mathcal{M}_{f}=\mathcal{M}_{T}$ para toda função contínua $f$.

\section{Demonstração.}

Como $f$ é uma função contínua, $f$ é limitada em $X$ compacto, ou seja, $f$ é limitada superiormente e inferiormente. Assim, segue o resultado da Observação anterior. 
Observação 2.1.5. Se $\mathcal{M}_{f}=\emptyset$, então $\alpha(f)=-\infty$. Em particular, se $\mathcal{M}_{T}=\emptyset$, então $\alpha(f)=-\infty$ para toda função contínua $f$.

Observação 2.1.6. Se $\mathcal{M}_{T} \neq \emptyset$, e $f \in \mathcal{L}^{1}(\mu)$ para alguma $\mu \in \mathcal{M}_{T}$, então $\alpha(f)>-\infty$.

\section{Demonstração.}

Como $f \in \mathcal{L}^{1}(\mu)$, temos que

$$
\begin{gathered}
\int_{X}|f| d \mu=\int_{X} f^{+} d \mu+\int_{X} f^{-} d \mu<\infty \\
\Rightarrow \int_{X} f^{+} d \mu<\infty \text { e } \int_{X} f^{-} d \mu<\infty .
\end{gathered}
$$

Assim,

$$
\begin{aligned}
\alpha(f) & =\sup _{m \in \mathcal{M}_{f}} \int_{X} f d m \geq \int_{X} f d \mu \\
& =\int_{X} f^{+} d \mu-\int_{X} f^{-} d \mu>-\infty .
\end{aligned}
$$

Observação 2.1.7. Se $f$ é mensurável e limitada superiormente, então $\alpha(f)<\infty$.

\section{Demonstração.}

Seja $c$ o limite superior de $f$. Logo,

$$
\int_{X} f^{+} d \mu \leq \int_{X} c d \mu=c \mu(X)=c<+\infty
$$

para $\mu \in \mathcal{M}_{T}$ qualquer.

Então,

$$
\begin{aligned}
\alpha(f) & =\sup _{\mu \in \mathcal{M}_{f}} \int_{X} f d \mu=\sup _{\mu \in \mathcal{M}_{f}}\left(\int_{X} f^{+} d \mu-\int_{X} f^{-} d \mu\right) \\
& \leq \sup _{\mu \in \mathcal{M}_{f}}\left(c-\int_{X} f^{-} d \mu\right) \leq c+\sup _{\mu \in \mathcal{M}_{f}}\left(-\int_{X} f^{-} d \mu\right) \leq c<+\infty .
\end{aligned}
$$


Observação 2.1.8. Se $f, g$ são contínuas e $f-g$ é limitada, então $\mathcal{M}_{f}=\mathcal{M}_{g}$.

\section{Demonstração.}

Como $f-g$ é limitada, $\int_{X} f-g d \mu$ existe para $\mu \in \mathcal{M}_{T}$.

Provemos que $\mathcal{M}_{f} \subset \mathcal{M}_{g}$.

Se $\mu \in \mathcal{M}_{f}$, então

$$
\begin{aligned}
\int_{X} f d \mu \text { existe } & \Rightarrow \int_{X} f-g d \mu-\int_{X} f d \mu \text { existe } \\
& \Rightarrow \int_{X}-g d \mu \text { existe } \\
& \Rightarrow \int_{X} g d \mu \text { existe } \\
& \Rightarrow \mu \in \mathcal{M}_{g} .
\end{aligned}
$$

$\mathcal{M}_{g} \subset \mathcal{M}_{f}$ é análogo.

Observação 2.1.9. Se $\delta(f) \in[-\infty, \infty)$, então $\sup _{x \in X} S_{n} f(x)$ é finito para todo $n$ suficientemente grande, e é uma sequência subaditiva de reais. Então, de fato, o limite $\lim _{n \rightarrow \infty} \frac{1}{n} \sup _{x \in X} S_{n} f(x) \in[-\infty, \infty)$ existe e é igual a $\inf _{n} \frac{1}{n} \sup _{x \in X} S_{n} f(x)$.

\section{Demonstração.}

Inicialmente, lembremos que, para uma sequência $x_{n}$,

$$
\lim \sup x_{n}=\inf _{n \geq 1}\left\{\sup \left\{x_{k}: k \geq n\right\}\right\} .
$$

Assim, 


$$
\begin{aligned}
\delta(f) & =\limsup _{n \rightarrow \infty} \frac{1}{n} \sup _{x \in X} S_{n} f(x)<\infty \\
& =\inf _{n \geq 1}\left\{\sup \left\{\frac{1}{k} \sup _{x \in X} S_{n} f(x): k \geq n\right\}\right\}<\infty \\
& \Rightarrow \exists n_{0}: \sup \left\{\frac{1}{k} \sup _{x \in X} S_{n} f(x): k \geq n_{0}\right\}<\infty \\
& \Rightarrow \forall k \geq n_{0}, \frac{1}{k} \sup _{x \in X} S_{n} f(x)<\infty \\
& \Rightarrow \forall k \geq n_{0}, \sup _{x \in X} S_{n} f(x)<\infty .
\end{aligned}
$$

Além disso,

$$
\begin{gathered}
S_{n+m} f(x)=\sum_{i=0}^{n+m-1} f \circ T^{i}(x)=\sum_{i=0}^{n-1} f \circ T^{i}(x)+\sum_{i=n}^{n+m-1} f \circ T^{i}(x) \\
=S_{n} f(x)+S_{m} f\left(T^{n}(x)\right) \\
\Rightarrow \sup _{x \in X} S_{n+m} f(x) \leq \sup _{x \in X} S_{n} f(x)+\sup _{x \in X} S_{m} f\left(T^{n}(x)\right) .
\end{gathered}
$$

Como $T^{n}(X) \subset X$, então

$$
\sup _{x \in X} S_{m} f\left(T^{n}(x)\right) \leq \sup _{x \in X} S_{m} f(x)
$$

donde,

$$
\sup _{x \in X} S_{n+m} f(x) \leq \sup _{x \in X} S_{n} f(x)+\sup _{x \in X} S_{m} f(x) .
$$

Logo, a sequência é subaditiva, e pelo lema 1.3.15:

o limite $\lim _{n \rightarrow \infty} \frac{1}{n} \sup _{x \in X} S_{n} f(x) \in[-\infty, \infty)$ existe e é igual a $\inf _{n} \frac{1}{n} \sup _{x \in X} S_{n} f(x)$. 


\subsection{Média ergódica máxima e medidas maximizantes}

Em geral, $\alpha(f), \beta(f), \gamma(f), \delta(f)$ não coincidem, embora possam ser impostas condições sobre $X$ e $T$ para que sejam iguais e então definir a média ergódica máxima.

Se $X$ é espaço métrico compacto não vazio, e $T: X \rightarrow X$ é contínua, então o conjunto $\mathcal{M}_{T}(X)$ é não vazio (1.2.6) e, quando equipado da topologia fraca-* é compacto (1.2.7). Estamos interessados nos casos nos quais $f$ é uma função característica de um subconjunto fechado ou é contínua.

Teorema 2.2.1. Seja $T: X \rightarrow X$ uma aplicação contínua num espaço métrico compacto. Se $f: X \rightarrow \mathbb{R}$ é contínua, ou uma função característica de um subconjunto fechado, então

$$
\alpha(f)=\beta(f)=\gamma(f)=\delta(f) \neq \pm \infty .
$$

\section{Demonstração.}

Primeiramente verificaremos as desigualdades $\alpha(f) \leq \beta(f) \leq \gamma(f) \leq \delta(f)$.

Observemos que, para essa parte da prova, $f$ pode ser contínua ou a função característica de um subconjunto fechado.

É imediato da definição que $\beta(f) \leq \gamma(f)$ e $\gamma(f) \leq \delta(f)$, pois:

$$
\begin{aligned}
\beta(f) & =\sup _{x \in \operatorname{Reg}(X, T, f)} \lim _{n \rightarrow \infty} \frac{1}{n} S_{n} f(x) \\
& \leq \sup _{x \in X} \limsup _{n \rightarrow \infty} \frac{1}{n} S_{n} f(x) \\
& =\gamma(f)
\end{aligned}
$$

e também

$$
\begin{aligned}
& \frac{1}{n} S_{n} f(x) \leq \frac{1}{n} \sup _{y \in X} S_{n} f(y) \\
\Rightarrow & \limsup _{n \rightarrow \infty} \frac{1}{n} S_{n} f(x) \leq \limsup _{n \rightarrow \infty} \frac{1}{n} \sup _{y \in X} S_{n} f(y) \\
\Rightarrow & \gamma(f)=\sup _{x \in X} \limsup _{n \rightarrow \infty} \frac{1}{n} S_{n} f(x) \leq \limsup _{n \rightarrow \infty} \frac{1}{n} \sup _{y \in X} S_{n} f(y)=\delta(f) .
\end{aligned}
$$


Assim, basta mostrar que $\alpha(f) \leq \beta(f)$.

Por hipótese, $X$ é compacto e $f$ é contínua, ou $f$ é característica de um subconjunto fechado, ou seja, $f$ é limitada em $X$. Então,pode-se aplicar o teorema de Birkhoff (1.3.1) para qualquer medida de probabilidade $T$-invariante.

Se $\mu \in M_{T}(X)$ é uma medida ergódica, para $\mu$-quase todo ponto $x \in X$,

$$
\int_{X} f d \mu=\lim _{n \rightarrow \infty} \frac{1}{n} S_{n} f(x) \leq \sup _{y \in \operatorname{Reg}(X, T, f)} \lim _{n \rightarrow \infty} \frac{1}{n} S_{n} f(y)=\beta(f) .
$$

Se $\mu \in M_{T}(X)$ é uma medida qualquer, usando 1.3 .13 e a desigualdade anterior:

$$
\int_{X} f d \mu=\int_{E \mathcal{M}_{T}(X)} \int_{X} f d m d \rho_{\mu}(m) \leq \int_{E \mathcal{M}_{T}(X)} \beta(f) d \rho_{\mu}(m)=\beta(f) .
$$

Em particular,

$$
\alpha(f)=\sup _{\mu \in \mathcal{M}_{f}} \int_{X} f d \mu \leq \beta(f)
$$

Logo, $\alpha(f) \leq \beta(f) \leq \gamma(f) \leq \delta(f)$.

Para provar a igualdade, é suficiente mostrar que $\alpha(f) \geq \delta(f)$.

Consideremos $\mu_{n}=\frac{1}{n} \sum_{i=0}^{n-1} \delta_{T^{i} x_{n}} \in \mathcal{M}(X)$, onde $x_{n}$ é tal que:

$$
\begin{aligned}
\frac{1}{n} \sup _{x \in X} S_{n} f(x) & =\frac{1}{n} S_{n} f\left(x_{n}\right) \\
& =\frac{1}{n} \sum_{i=0}^{n-1} f \circ T^{i}\left(x_{n}\right) \\
& =\int_{X} \frac{1}{n} \sum_{i=0}^{n-1} f d \delta_{T^{i} x_{n}}=\int_{X} f d \mu_{n} .
\end{aligned}
$$

Como $\mathcal{M}(X)$ é compacto (1.2.5), existe subsequência $\mu_{n_{k}}$ tal que $\mu_{n_{k}} \rightarrow \bar{\mu}$, com $\bar{\mu} \in \mathcal{M}(X)$.

Por argumento análogo ao da prova de 1.2.6, pode-se mostrar que na verdade $\bar{\mu}$ é $T$-invariante, ou seja, $\bar{\mu} \in \mathcal{M}_{T}$.

- Se f é contínua: 
Pela igualdade acima e pela definição de convergência fraca,

$$
\lim _{n_{k} \rightarrow \infty} \frac{1}{n_{k}} \sup _{x \in X} S_{n_{k}} f(x)=\lim _{n_{k} \rightarrow \infty} \int_{X} f d \mu_{n_{k}}=\int_{X} f d \bar{\mu} .
$$

Assim,

$$
\limsup _{n_{k} \rightarrow \infty} \int_{X} f d \mu_{n_{k}}=\int_{X} f d \bar{\mu}
$$

e

$$
\begin{aligned}
\delta(f) & =\limsup _{n_{k} \rightarrow \infty} \frac{1}{n_{k}} \sup _{x \in X} S_{n_{k}} f(x)=\limsup _{n_{k} \rightarrow \infty} \int_{X} f d \mu_{n_{k}} \\
& =\int_{X} f d \mu \leq \sup _{m \in \mathcal{M}_{f}} \int_{X} f d m=\alpha(f) .
\end{aligned}
$$

- Se f é função característica de um subconjunto fechado $F$ :

$$
\begin{aligned}
\delta(f) & =\limsup _{n \rightarrow \infty} \frac{1}{n} \sup _{x \in X} S_{n} f(x) \stackrel{2.2}{=} \limsup _{n \rightarrow \infty} \int_{X} f d \mu_{n} \\
& =\limsup _{n \rightarrow \infty} \mu_{n}(F) \stackrel{1.2 .8}{\leq} \bar{\mu}(F)=\int_{X} f d \bar{\mu} \leq \alpha(f) .
\end{aligned}
$$

Além disso, em ambos os casos, $f$ é limitada em $X$. Pela observação 2.1.6, $\alpha(f)>-\infty$. Analogamente, $\alpha(f)<\infty$.

Portanto,

$$
\alpha(f)=\beta(f)=\gamma(f)=\delta(f) \neq \pm \infty .
$$

A prova do teorema anterior sugere uma generalização. Para isso, lembremos dos seguintes conceitos encontrados em [2]:

Definição 2.2.2. Seja $f: X \rightarrow \mathbb{R}$ uma função, onde $X$ é um espaço topológico. Tal função é dita semi-contínua superiormente em $x_{0} \in X$ quando, dado $\epsilon>0$, existe $\delta>0$ tal que, se $\left|x-x_{0}\right|<\delta$, então

$$
f(x) \leq f\left(x_{0}\right)+\epsilon
$$


Equivalentemente,

$$
\limsup _{x \rightarrow x_{0}} f(x) \leq f\left(x_{0}\right)
$$

Teorema 2.2.3. Seja $f: E \rightarrow[-\infty, \infty)$. Se E é compacto e $f$ é uma função semicontínua superiormente então $f$ alcança sua cota superior.

Teorema 2.2.4. Seja $T: X \rightarrow X$ uma aplicação contínua num espaço métrico compacto. Se $f: X \rightarrow \mathbb{R}$ é semi-contínua superiormente, então

$$
\alpha(f)=\beta(f)=\gamma(f)=\delta(f) \in[-\infty, \infty)
$$

\section{Demonstração.}

Analogamente a 2.2.1, $\alpha(f) \leq \beta(f) \leq \gamma(f) \leq \delta(f)$. Basta verificar que para usar o teorema de Birkhoff, na prova em que $\alpha(f) \leq \beta(f)$, podemos supor $\int_{X} f d \mu>-\infty$, pois senão:

$$
\int_{X} f d \mu=-\infty, \forall \mu \in \mathcal{M}_{f} \Rightarrow \alpha(f)=-\infty \leq \beta(f) .
$$

E como $f$ é semi-contínua superiormente em $X$ compacto, usando 2.2.3, $\int_{X} f d \mu<\infty$.

Falta mostrar que $\delta(f) \leq \alpha(f)$. Essa parte também é análoga, observando que basta conseguir uma desigualdade em 2.3 e seguirá o restante.

Para isso considere a aplicação $F: \mathcal{M}(X) \rightarrow[-\infty, \infty)$ definida da seguinte maneira:

$$
F(\mu)=\int_{X} f d \mu
$$

$F(\mu) \in[-\infty, \infty)$ para toda $\mu \in \mathcal{M}(X)$ pois $f$ é limitada superiormente.

Mostremos que $F$ é semi-contínua superiormente, ou seja, se $\mu_{n} \rightarrow \mu \in \mathcal{M}(X)$, então

$$
\int_{X} f d \mu \geq \limsup _{n \rightarrow \infty} \int_{X} f d \mu_{n}
$$

como o que queríamos. 
Iniciemos utilizando uma equivalência de semi-continuidade superior para $f$. Existe subsequência de funções contínuas $f_{i}: X \rightarrow \mathbb{R}$ com $f_{i} \geq f_{i+1}$, para todo $i$ tal que,

$$
\lim _{i \rightarrow \infty} f_{i}(x)=f(x)
$$

- Se $\int_{X} f d \mu>-\infty$,

tome $g_{i}=f-f_{i}$, implicando que $g_{i} \leq g_{i+1}$ e $g_{i} \rightarrow 0$. Pelo Teorema da convergência monótona (1.1.3),

$$
\lim _{i \rightarrow \infty} \int_{X} f-f_{i} d \mu=0
$$

Logo, dado $\epsilon>0, \int_{X} f-f_{i} d \mu>-\epsilon$ para $i$ suficientemente grande, e

$$
\begin{aligned}
\int_{X} f d \mu & =\int_{X} f-f_{i} d \mu+\int_{X} f_{i} d \mu \\
& >-\epsilon+\int_{X} f_{i} d \mu \\
& =-\epsilon+\limsup _{n \rightarrow \infty} \int_{X} f_{i} d \mu_{n} \\
& \geq-\epsilon+\limsup _{n \rightarrow \infty} \int_{X} f d \mu_{n}
\end{aligned}
$$

ou seja,

$$
\int_{X} f d \mu \geq \limsup _{n \rightarrow \infty} \int_{X} f d \mu_{n}
$$

- Se $\int_{X} f d \mu=-\infty$,

para todo $j \in \mathbb{N}$,

$$
\limsup _{n \rightarrow \infty} \int_{X} f d \mu_{n} \leq \limsup _{n \rightarrow \infty} \int_{X} \max \{f,-j\} d \mu_{n} \stackrel{2.4}{\leq} \int_{X} \max \{f,-j\} d \mu .
$$

Fazendo $j \rightarrow \infty$, temos que

$$
\limsup _{n \rightarrow \infty} \int_{X} f d \mu_{n}=-\infty .
$$


Portanto, vale a desigualdade em 2.3, e o teorema está provado.

Definição 2.2.5. Seja $X$ um espaço topológico, e suponha que $T: X \rightarrow X$ é contínua. A quantidade

$$
\alpha(f)=\sup _{m \in \mathcal{M}_{f}} \int_{X} f d \mu
$$

é chamada de média ergódica máxima para $f$. Uma medida $\mu \in \mathcal{M}_{T}$ é chamada $f$ maximizante se $\alpha(f)=\int_{X} f d \mu$, e o conjunto de todas as medidas maximizantes é denotado por $\mathcal{M}_{\max }(f)$.

\subsection{O conjunto das medidas maximizantes}

O objeto de interesse da otimização ergódica são essas medidas maximizantes. Daí, segue a importância de estudar a existência dessas medidas e a caracterização de $\mathcal{M}_{\text {max }}(f)$.

Teorema 2.3.1. Se $X$ é um espaço métrico compacto e $f$ é contínua, então $\mathcal{M}_{\max }(f) \neq \emptyset$.

\section{Demonstração.}

Considere $F: \mathcal{M}_{T}(X) \rightarrow(-\infty, \infty)$ definida por $F(\mu)=\int_{X} f d \mu$. Essa função é contínua na topologia fraca-*

De fato, seja $\mu_{n} \rightarrow \mu$ em $\mathcal{M}_{T}(X)$. Por 1.2.3,

$$
\int_{X} g d \mu_{n} \rightarrow \int_{X} g d \mu
$$

para toda $g \in \mathcal{C}^{0}(X)$. Em particular,

$$
F\left(\mu_{n}\right) \rightarrow F(\mu)
$$

Além disso, como $X$ é um espaço métrico compacto, $\mathcal{M}_{T}(X)$ é compacto (1.2.7). 
Assim, segue que essa função assume máximo em $\mathcal{M}_{T}(X)$.

Abaixo, segue a generalização:

Teorema 2.3.2. Seja $T: X \rightarrow X$ uma aplicação contínua num espaço métrico compacto e suponha que $f: X \rightarrow \mathbb{R}$ é semi-contínua superiormente. Então

i) $\mathcal{M}_{\max }(f) \neq \emptyset$.

ii) $\mathcal{M}_{\text {max }}(f)$ é convexo e compacto.

\section{Demonstração.}

i) Considere $F: \mathcal{M}_{T}(X) \rightarrow[-\infty, \infty)$ como definida em 2.2.4. Essa função é semicontínua superiormente e $\mathcal{M}_{T}(X)$ é compacto. Por 2.2.3, $F$ assume máximo em $M_{T}(X)$ e, portanto $\mathcal{M}_{\max }(f) \neq \emptyset$.

ii) Mostremos que $\mathcal{M}_{\max }(f)$ é convexo, ou seja, se para quaisquer $\mu_{1}, \mu_{2} \in \mathcal{M}_{\max }(f)$ e para qualquer $t \in[0,1]$,

$$
\mu_{t}=(1-t) \mu_{1}+t \mu_{2} \in \mathcal{M}_{\max }(f) .
$$

De fato, como $\mu_{1}, \mu_{2} \in \mathcal{M}_{\max }(f)$ então $\int_{X} f d \mu_{1}=\int_{X} f d \mu_{2}=\sup _{m \in \mathcal{M}_{f}} \int_{X} f d m$. Logo,

$$
\begin{aligned}
\int_{X} f d \mu_{t} & =\int_{X} f d\left((1-t) \mu_{1}\right)+\int_{X} f d\left(t \mu_{2}\right) \\
& =(1-t) \int_{X} f d \mu_{1}+t \int_{X} f d \mu_{1}=\sup _{m \in \mathcal{M}_{f}} \int_{X} f d m .
\end{aligned}
$$

Mostremos que $\mathcal{M}_{T}(X)$ é fechado, ou seja, se $\mu_{n} \in \mathcal{M}_{\text {max }}(f) \operatorname{com} \mu_{n} \rightarrow \mu$, então $\mu \in \mathcal{M}_{T}(X)$.

Como $\mu_{n} \in \mathcal{M}_{T}(X)$ e $\mathcal{M}_{T}(X)$ é fechado então $\mu \in \mathcal{M}_{T}(X)$, ou seja,

$$
\int_{X} f d \mu \leq \sup _{m \in \mathcal{M}_{f}} \int_{X} f d m .
$$


Também, como a função $F$ considerada anteriormente é semi-contínua superiormente:

$$
\int_{X} f d \mu \geq \limsup _{n \rightarrow \infty} \int_{X} f d \mu_{n} \geq \int_{X} f d \mu_{n}=\sup _{m \in \mathcal{M}_{f}} \int_{X} f d m
$$

Portanto,

$$
\int_{X} f d \mu=\sup _{m \in \mathcal{M}_{f}} \int_{X} f d m
$$

Além disso, como $\mathcal{M}_{T}(X)$ é compacto, então $\mathcal{M}_{\text {max }}(f) \subset \mathcal{M}_{T}(X)$ é compacto.

Com respeito a caracterização do conjunto das medidas maximizantes, temos o seguinte resultado:

Teorema 2.3.3. O conjunto $\left\{\mathcal{M}_{\max }(f): f \in \mathcal{C}(X)\right\}$ é precisamente o conjunto das faces $^{1}$ fechadas de $\mathcal{M}_{T}$.

A demonstração pode ser encontrada em [9].

\footnotetext{
${ }^{1}$ Um subconjunto convexo e não-vazio $F \subset K$ é chamado de face de $K$ se, quando $\lambda k_{1}+(1-\lambda) k_{2} \in F$ com $k_{1}, k_{2} \in K$ e $0<\lambda<1$, então $k_{1}, k_{2} \in F$.
} 


\section{CAPÍTULO 3}

\section{— Otimização ergódica para sistemas dinâmicos não-compactos}

Iniciaremos o estudo da otimização ergódica para sistemas dinâmicos topológicos gerais $T: X \rightarrow X$, onde o espaço topológico $X$ não é necessariamente compacto. Se $f: X \rightarrow X$ é contínua, apresentaremos condições que garantem a existência de medidas $f$-maximizantes, as quais serão caracterizadas pelo seu suporte. Esses são resultados de $[6]$.

\subsection{Introdução}

Seja $X$ um espaço topológico, não necessariamente compacto. No capítulo 1 vimos que para o caso em que $X$ é compacto, $\mathcal{M}_{T} \neq \emptyset$ (1.2.6). Mas, em geral, $\mathcal{M}_{T}$ pode ser vazio, como mostra o exemplo seguinte:

Exemplo 3.1.1. Seja $X=\left\{\frac{1}{n}, n \in \mathbb{N}^{*}\right\}$ e $T: X \rightarrow X$ tal que $T\left(\frac{1}{n}\right)=\frac{1}{n-1}, n>1$.

Suponhamos que exista $\mu \in \mathcal{M}_{T}(X)$. Então:

$$
\mu\left(\frac{1}{n}\right)=\mu\left(\frac{1}{n+1}\right), n \geq 1
$$


Mas,

$$
\mu(X)=\sum_{n \in \mathbb{N}} \mu\left(\frac{1}{n}\right)=\sum_{n \in \mathbb{N}} c,
$$

onde $c \in \mathbb{N}$ é constante.

Se $c=0$ então $\mu(X)=0$.

Se $c>0$ então $\mu(X)=\infty$.

Absurdo, pois a medida é de probabilidade. Logo, $\mathcal{M}_{T}(X)=\emptyset$.

A fim de estudar espaços mais abrangentes que os compactos, definimos:

Definição 3.1.2. Um espaço polonês é um espaço topológico completamente metrizável e separável.

Para o próximo resultado, precisaremos dos seguintes lema e teorema vistos respectivamente em [11] e [4]:

Lema 3.1.3. Se T é um endomorfismo ergódico de um espaço de probabilidade $(X, \mathcal{B}, \mu)$, $f \geq 0 e \int_{X} f d \mu=\infty$, então o teorema de Birkhoff implica $\lim _{n \rightarrow \infty} \frac{1}{n} S_{n} f=\infty$.

Teorema 3.1.4. Sejam $X$ um espaço polonês e $T: X \rightarrow X$ uma função contínua. Então, para cada $\mu \in \mathcal{M}_{T}(X)$, existe $\rho_{\mu}$ uma medida definida sobre os borelianos de $\mathcal{M}_{T}(X)$, com a propriedade que $\rho_{\mu}\left(\operatorname{E\mathcal {M}}_{T}(X)\right)=1 e$

$$
\mu=\int_{E \mathcal{M}_{T}(X)} m d \rho_{\mu}(m),
$$

onde $\operatorname{EM}_{T}(X)$ são as medidas ergódicas em $\mathcal{M}_{T}(X)$.

Teorema 3.1.5. Seja $X$ um espaço polonês. Se $T: X \rightarrow X$ e $f: X \rightarrow \mathbb{R}$ são continuas, então $\alpha(f) \leq \beta(f) \leq \gamma(f) \leq \delta(f)$. 
Demonstração. Observemos que bastam alguns ajustes na primeira parte de 2.2.1 para analogamente provar a desigualdade:

$$
\alpha(f) \leq \beta(f) \leq \gamma(f) \leq \delta(f)
$$

Como tínhamos que $X$ era compacto, utilizamos a limitação de $f$ no teorema ergódico de Birkhoff na prova de $\alpha(f) \leq \beta(f)$. Aqui, como não temos essa hipótese, dividimos em dois casos:

Se $f \in \mathcal{L}^{1}(\mu)$, vale o teorema de Birkhoff (1.3.1).

Se $f \notin \mathcal{L}^{1}(\mu)$, como $\int_{X}|f| d \mu=\infty$, usamos o lema 3.1.3. Observe que se $f$ assume valores negativos, basta considerar $f=f^{+}-f^{-}$e trabalhar com as funções não-negativas $f^{+}$e $f^{-}$. Como $f \in \mathcal{M}_{f}, \int_{X} f^{+} d \mu<\infty$ ou $\int_{X} f^{-} d \mu<\infty$, e usando o teorema de Birkhoff para uma dessas funções e o lema para a outra, chegamos ao mesmo resultado.

Além disso, utilizamos a decomposição ergódica no espaço $X$ compacto. A generalização desse teorema para um espaço polonês é dada em 3.1.4, e o restante da demonstração será análoga.

O próximo exemplo mostra que as desigualdades do teorema anterior podem ser estritas.

Exemplo 3.1.6. Consideremos $\mathbb{Z}$ com a topologia discreta.

$$
\text { Defina } T: \mathbb{Z} \rightarrow \mathbb{Z} \text { por } T(i)=\left\{\begin{array}{l}
i+1, \text { se } i<0 \\
i, \text { se } i=0 \\
i+2, \text { se } i>0
\end{array}\right.
$$


Defina $f: \mathbb{Z} \rightarrow \mathbb{R}$ por $f(i)=\left\{\begin{array}{l}2, \text { se } i<0 \\ -1, \text { se } i=0 \\ 0, \text { se } i>0 \text { é ímpar } \\ \frac{i}{2}, \text { se } i>0 \text { e } i \equiv 2(\bmod 4) \\ 1-\frac{i}{2}, \text { se } i>0 \text { e } i \equiv 0(\bmod 4)\end{array}\right.$

Para encontrar o valor de $\alpha(f)$, vejamos quem são as medidas $\mu \in \mathcal{M}_{T}$.

Se $i<0, \mu(i)=\mu(i-1)$, ou seja, $\mu(i)=c$, onde $c \geq 0$ é constante. Como a medida é de probabilidade, resulta que $\mu(i)=0$.

Se $i>0, \mu(i)=\mu(i-2)$, e analogamente $\mu(i)=0$.

Se $i=0, \mu(i)=\mu\left(T^{-1}(i)\right)=1$, pois a medida é de probabilidade.

Logo, a única medida T-invariante é a medida de Dirac concentrada no zero, donde

$$
\alpha(f)=\sup _{m \in \mathcal{M}_{f}} \int_{\mathbb{Z}} f d m=\int_{\mathbb{Z}} f d \delta_{0}=f(0)=-1
$$

Para encontrar os outros valores, analisemos $S_{n} f(i)$ para cada $i \in \mathbb{Z}$ :

- $i>0$ :

$$
S_{n} f(i)=\sum_{j=0}^{n-1} f \circ T^{j}(i)=\sum_{j=0}^{n-1} f(i+2 j) .
$$

1. $i$ impar

$$
S_{n} f(i)=f(i)+f(\underbrace{i+2}_{\text {impar }})+\ldots+f(\underbrace{i+2 n-2}_{\text {ímpar }})=0+0+\ldots+0=0 .
$$

2. $i \equiv 2(\bmod 4)$

Para $n$ impar,

$$
\begin{aligned}
S_{n} f(i) & =f(i)+f \underbrace{(i+2)}_{i \equiv 0(\bmod 4)}+\ldots+f(\underbrace{i+2 n-2}_{i \equiv 2(\bmod 4)}) \\
& =\frac{i}{2}+\left(1-\frac{i+2}{2}\right)+\ldots+\left(\frac{i}{2}+n-1\right) \\
& =0+\ldots+\left(\frac{i}{2}+n-1\right)=\frac{i}{2}+n-1 .
\end{aligned}
$$


Para $n$ par,

$$
\begin{aligned}
S_{n} f(i) & =f(i)+f \underbrace{(i+2)}_{i \equiv 0(\bmod 4)}+\ldots+f(\underbrace{i+2 n-4}_{i \equiv 2(\bmod 4)})+f(\underbrace{i+2 n-2}_{i \equiv 0(\bmod 4)}) \\
& =\frac{i}{2}+\left(1-\frac{i+2}{2}\right)+\ldots+\left(\frac{i}{2}+n-2\right)+\left(1-\frac{i+2 n-2}{2}\right) \\
& =0+\ldots+0=0 .
\end{aligned}
$$

Assim, não existe $\lim _{n \rightarrow \infty} \frac{1}{n} S_{n} f(i)$, ou seja, $i \notin \operatorname{Reg}(\mathbb{Z}, T, f)$.

3. $i \equiv 0 \quad(\bmod 4)$

Para $n$ ímpar,

$$
\begin{aligned}
S_{n} f(i) & =f(i)+f \underbrace{(i+2)}_{i \equiv 2(\bmod 4)}+\ldots+f(\underbrace{i+2 n-2}_{i \equiv 0(\bmod 4)}) \\
& =\left(1-\frac{i}{2}\right)+\left(\frac{i+2}{2}\right)+\ldots+\left(1-\frac{i+2 n-2}{2}\right) \\
& =2+\cdots+\left(2-\frac{i}{2}-n\right)=(n-1)+\left(2-\frac{i}{2}-n\right)=-\frac{i}{2}+1 .
\end{aligned}
$$

Para $n$ par,

$$
\begin{aligned}
S_{n} f(i) & =f(i)+f \underbrace{(i+2)}_{i \equiv 2(\bmod 4)}+\ldots+f(\underbrace{i+2 n-4}_{i \equiv 0(\bmod 4)})+f(\underbrace{i+2 n-2}_{i \equiv 2(\bmod 4)}) \\
& =\left(1-\frac{i}{2}\right)+\left(\frac{i+2}{2}\right)+\ldots+\left(1-\frac{i+2 n-4}{2}\right)+\left(\frac{i+2 n-2}{2}\right) \\
& =2+\cdots+2=n .
\end{aligned}
$$

Assim, não existe $\lim _{n \rightarrow \infty} \frac{1}{n} S_{n} f(i)$, ou seja, $i \notin \operatorname{Reg}(\mathbb{Z}, T, f)$.

- $i<0$ :

$$
\begin{aligned}
S_{n} f(i) & =\sum_{j=0}^{n-1} f \circ T^{j}(i)=f(i)+\ldots+f(-1)+f(0)+\ldots+f(0) \\
& =2+\ldots+2+(-1)+\ldots+(-1) \\
& =2(-i)+(n-(-i))(-1)=-3 i-n .
\end{aligned}
$$


- $i=0$ :

$$
S_{n} f(i)=\sum_{j=0}^{n-1} f \circ T^{j}(0)=\sum_{j=0}^{n-1} f(0)=-n .
$$

Implicando que,

$$
\begin{aligned}
& \beta(f)=\sup _{i \in \operatorname{Reg}(\mathbb{Z}, T, f)} \lim _{n \rightarrow \infty} \frac{1}{n} S_{n} f(i)=\max \{-1,0\}=0 \\
& \gamma(f)=\sup _{i \in \mathbb{Z}} \limsup _{n \rightarrow \infty} \frac{1}{n} S_{n} f(i)=\max \{-1,0,1\}=1
\end{aligned}
$$

Finalizando, para $i=-n<0, S_{n} f(i)=-3(-n)-n=2 n$.

Então,

$$
\delta(f)=\limsup _{n \rightarrow \infty} \frac{1}{n} \sup _{i \in \mathbb{Z}} S_{n} f(i)=2
$$

Já vimos que a compacidade de $X$ garante a existência de medida maximizante (2.3.1). O próximo exemplo mostra que $\mathcal{M}_{\max }(f)$ pode ser vazio, mesmo quando $\mathcal{M}_{f} \neq \emptyset$.

Exemplo 3.1.7. Sejam $T: \mathbb{R} \rightarrow \mathbb{R}$ definida por $T(x)=x$ e $f: \mathbb{R} \rightarrow \mathbb{R}$ definida por $f(x)=1-e^{-x}$.

Primeiramente, observemos que toda medida $\mu$ de probabilidade é T-invariante.

Como $f$ é limitada superiormente, usando a observação 2.1.3, $\mathcal{M}_{f}=\mathcal{M}_{T} \neq \emptyset$, como queríamos.

Temos também que $\int_{\mathbb{R}} 1-e^{-x} d \mu<\int_{\mathbb{R}} 1 d \mu=\mu(\mathbb{R})=1$, ou seja, 1 é uma cota superior de $\int_{\mathbb{R}} f d \mu$. Mostremos que é a menor.

Dado $\epsilon>0$, existe $\delta_{p}$, medida de Dirac concentrada no ponto $p$, tal que

$$
1-\epsilon<\int_{\mathbb{R}} 1-e^{-x} d \delta_{p}=f(p)=1-e^{-p}<1 .
$$

Portanto,

$$
\alpha(f)=\sup _{\mu \in \mathcal{M}_{f}} \int_{\mathbb{R}} f d \mu=1
$$


Entretanto, como $\int_{\mathbb{R}} f d \mu<1$, não existe $\mu \in \mathcal{M}_{f}$ tal que $\int_{\mathbb{R}} 1-e^{-x} d \mu=\alpha(f)$.

\subsection{Forma normal e caracterização das medidas ma- ximizantes}

Consideraremos o conjunto das medidas f-maximizantes onde $\alpha(f)=\delta(f) \neq \pm \infty$. Para estudar e entender esse conjunto, tentaremos modificar a função $f$.

Denotemos por $\mathcal{C B}(X)$ o conjunto das funções contínuas em $X$ e limitadas por valores reais.

Definição 3.2.1. Para uma função contínua $T: X \rightarrow X$, uma função da forma $\varphi-\varphi \circ T$, onde $\varphi \in \mathcal{C B}(X)$, é chamada função cobordo.

Definição 3.2.2. Duas funções $f, g$ são chamadas cohomólogas se a diferença entre elas é dada por uma função cobordo.

Denotamos por $f \sim g$.

Os resultados seguintes mostram a importância de considerar funções cohomólogas no estuda da otimização ergódica:

Lema 3.2.3. Suponha $T: X \rightarrow X$ uma função contínua no espaço topológico $X, e$ a função contínua $f: X \rightarrow \mathbb{R}$ cohomóloga a $\tilde{f}$. Então, $\int_{X} f d m=\int_{X} \tilde{f} d m$, para $m \in \mathcal{M}_{T}$ qualquer.

\section{Demonstração.}

$$
\int_{X} f-\tilde{f} d m=\int_{X} \varphi d m-\int_{X} \varphi \circ T d m=0 \quad(\text { por 1.1.4), }
$$


onde $\varphi \in \mathcal{C B}(X)$.

Corolário 3.2.4. Se $T: X \rightarrow X$ é uma função contínua no espaço topológico $X e$ $f: X \rightarrow \mathbb{R}$ é contínua com uma função cohomóloga $\tilde{f}$, então $\alpha(f)=\alpha(\tilde{f})$. Mais do que isso, $\alpha(f)<\sup \tilde{f}$.

\section{Demonstração.}

$$
\alpha(f)=\sup _{m \in \mathcal{M}_{f}} \int_{X} f d m=\sup _{m \in \mathcal{M}_{f}} \int_{X} \tilde{f} d m=\alpha(\tilde{f}) \leq \sup \tilde{f}
$$

Lema 3.2.5. Seja $T: X \rightarrow X$ uma função contínua no espaço topológico $X$. Considere $\tilde{f}$ cohomóloga a função contínua $f: X \rightarrow \mathbb{R}$. Então,

$$
\beta(f)=\beta(\tilde{f}), \gamma(f)=\gamma(\tilde{f}) \text { e } \delta(f)=\delta(\tilde{f})
$$

Demonstração. Seja $\varphi \in \mathcal{C B}(X)$. Então,

$$
\begin{aligned}
\beta(\tilde{f}) & =\sup _{x \in \operatorname{Reg}(X, T, f)} \lim _{n \rightarrow \infty} \frac{1}{n} S_{n} \tilde{f}(x) \\
& =\sup _{x \in \operatorname{Reg}(X, T, f)} \lim _{n \rightarrow \infty} \frac{1}{n} \sum_{i=0}^{n-1}(f+\varphi-\varphi \circ T)\left(T^{i}(x)\right) \\
& =\sup _{x \in \operatorname{Reg}(X, T, f)} \lim _{n \rightarrow \infty} \frac{1}{n}\left(\sum_{i=0}^{n-1} f\left(T^{i}(x)\right)+\sum_{i=0}^{n-1} \varphi\left(T^{i}(x)\right)-\sum_{i=1}^{n} \varphi\left(T^{i}(x)\right)\right) \\
& =\sup _{x \in \operatorname{Reg}(X, T, f)} \lim _{n \rightarrow \infty} \frac{1}{n}(\sum_{i=0}^{n-1} f\left(T^{i}(x)\right)+\underbrace{\varphi(x)-\varphi\left(T^{n}(x)\right)}_{\text {constante }}) \\
& =\sup _{x \in \operatorname{Reg}(X, T, f)} \lim _{n \rightarrow \infty} \frac{1}{n}\left(\sum_{i=0}^{n-1} f\left(T^{i}(x)\right)\right)=\beta(f) .
\end{aligned}
$$


A prova para $\gamma(f)$ e $\delta(f)$ são análogas.

Dentro do conjunto das funções cohomólogas, procuramos aquela que melhor caracteriza $\mathcal{M}_{\max }$.

Definição 3.2.6. Uma função contínua $\tilde{f} \sim f$ é chamada uma forma normal para $f$ se $\tilde{f}^{-1}(\sup \tilde{f}) \supset \operatorname{supp}(\mu)$, para alguma medida $\mu$ T-invariante.

Lema 3.2.7. Se $T: X \rightarrow X$ é uma função contínua no espaço topológico $X$ e $f: X \rightarrow \mathbb{R}$ é uma função contínua que tem uma forma normal $\tilde{f}$, então

$$
\alpha(f)=\sup \tilde{f}
$$

Demonstração. Como $f$ e $\tilde{f}$ são cohomólogas, por 3.2.4, $\alpha(f) \leq \sup \tilde{f}$.

Além disso, se $\mu \in \mathcal{M}_{T}$ satisfaz $\operatorname{supp}(\mu) \subset \tilde{f}^{-1}(\sup \tilde{f})$, então:

$$
\begin{aligned}
\int_{X} f d \mu & =\int_{X} \tilde{f} d \mu=\int_{\tilde{f}^{-1}(\sup \tilde{f})} \tilde{f} d \mu=\int_{X} \tilde{f} \cdot \chi_{\tilde{f}^{-1}(\sup \tilde{f})} d \mu \\
& =\int_{X} \sup \tilde{f} d \mu=\sup \tilde{f}<\infty .
\end{aligned}
$$

Logo, $\alpha(f)=\sup \tilde{f}$. Mais do que isso, $\mu$ é medida maximizante.

Lema 3.2.8. Seja $T: X \rightarrow X$ uma função contínua no espaço topológico $X$. Considere $\tilde{f}$ uma forma normal para a função contínua $f: X \rightarrow \mathbb{R}$. Então,

$$
\alpha(f)=\beta(f)=\gamma(f)=\delta(f)
$$

Demonstração. Mostremos que $\alpha(f) \leq \beta(f)$ :

Seja $\bar{x} \in \operatorname{supp}(\mu) . \operatorname{Então} T^{i}(\bar{x}) \in \operatorname{supp}(\mu) \subset \tilde{f}^{-1}(\sup \tilde{f})$ e

$$
\lim _{n \rightarrow \infty} \frac{1}{n} \sum_{i=0}^{n-1} \tilde{f}\left(T^{i}(\bar{x})\right)=\lim _{n \rightarrow \infty} \frac{1}{n} \sum_{i=0}^{n-1} \sup \tilde{f}=\sup \tilde{f}<\infty .
$$


Assim, $\bar{x} \in \operatorname{Reg}(X, T, f)$ e consequentemente

$$
\begin{aligned}
\beta(f)=\beta(\tilde{f}) & =\sup _{x \in \operatorname{Reg}(X, T, f)} \lim _{n \rightarrow \infty} \frac{1}{n} \sum_{i=0}^{n-1} S_{n} \tilde{f}(x) \\
& \geq \lim _{n \rightarrow \infty} \frac{1}{n} \sum_{i=0}^{n-1} \tilde{f}\left(T^{i}(\bar{x})\right)=\sup \tilde{f}=\alpha(f) .
\end{aligned}
$$

Analogamente ao feito em 2.2.1, pela definição temos também que $\beta(f) \leq \gamma(f)$ e $\gamma(f) \leq \delta(f)$.

Por fim, $\delta(f) \leq \alpha(f)$ :

$$
\begin{aligned}
\delta(f)=\delta(\tilde{f}) & =\limsup _{n \rightarrow \infty} \frac{1}{n} \sup _{x \in X} S_{n} \tilde{f}(x) \\
& \leq \limsup _{n \rightarrow \infty} \frac{1}{n} \sup _{x \in X} \sum_{i=0}^{n-1} \sup \tilde{f}=\sup \tilde{f}=\alpha(f) .
\end{aligned}
$$

O próximo teorema mostra que, se $f$ tem uma forma normal, suas medidas maximizantes são caracterizadas pelo seu suporte.

Teorema 3.2.9. Suponha $T: X \rightarrow X$ uma função contínua no espaço topológico $X$. Assuma que a função contínua $f: X \rightarrow \mathbb{R}$ tem uma forma normal $\tilde{f}$. Então $\mathcal{M}_{f}=\mathcal{M}_{T}$ $e$

$$
\mathcal{M}_{\text {max }}(f)=\left\{\mu \in \mathcal{M}_{T}: \operatorname{supp}(\mu) \subset \tilde{f}^{-1}(\sup \tilde{f})\right\} \neq \emptyset
$$

\section{Demonstração.}

Pela própria definição de forma normal, $\tilde{f}$ é limitada superiormente, e de 2.1.3, resulta que $\mathcal{M}_{\tilde{f}}=\mathcal{M}_{T}$. Além disso, $\tilde{f} \sim f$, ou seja, $\tilde{f}-f \in \mathcal{C B}(X)$; pela observação 2.1.8, $\mathcal{M}_{f}=\mathcal{M}_{\tilde{f}}=\mathcal{M}_{T}$.

Como $\tilde{f}$ é uma forma normal de $f$, a própria definição garante que

$$
\left\{\mu \in \mathcal{M}_{T}: \operatorname{supp}(\mu) \subset \tilde{f}^{-1}(\sup \tilde{f})\right\} \neq \emptyset
$$

Por 3.2.7, temos que $\alpha(f)=\sup \tilde{f}$ e $\mu$ é uma medida maximizante. Assim,

$$
\mathcal{M}_{\text {max }}(f) \supset\left\{m \in \mathcal{M}_{T}: \operatorname{supp}(m) \subset \tilde{f}^{-1}(\sup \tilde{f})\right\} .
$$


Mostremos agora que $\mathcal{M}_{\text {max }}(f) \subset\left\{m \in \mathcal{M}_{T}: \operatorname{supp}(m) \subset \tilde{f}^{-1}(\sup \tilde{f})\right\}$.

Seja $\mu \in \mathcal{M}_{\text {max }}$. Como sup $\tilde{f}-\tilde{f} \geq 0$ e

$$
0=\alpha(f)-\int_{X} f d \mu=\sup \tilde{f}-\int_{X} \tilde{f} d \mu=\int_{X} \sup \tilde{f}-\tilde{f} d \mu
$$

necessariamente $(\sup \tilde{f}-\tilde{f})=0 \mu$-q.t.p., donde $\operatorname{supp}(\mu) \subset \tilde{f}^{-1}(\sup \tilde{f})$.

Nem toda função contínua tem uma forma normal, como mostra o corolário do próximo teorema, provado em [3].

Teorema 3.2.10. Considere $T: \mathbb{R} / \mathbb{Z} \rightarrow \mathbb{R} / \mathbb{Z}$ definida por $T(x)=2 x(\bmod 1)$, ou seja, a função que duplica o ângulo no círculo. Para uma função $f \in \mathcal{C}^{0}(R / \mathbb{Z})$ genérica ${ }^{1}$, toda medida maximizante de $f$ tem suporte total.

Corolário 3.2.11. Seja $T: \mathbb{R} / \mathbb{Z} \rightarrow \mathbb{R} / \mathbb{Z}$ definida por $T(x)=2 x(\bmod 1)$. Para uma $f \in C^{0}(R / \mathbb{Z})$ genérica, não existe função não-positiva cohomóloga a $f-\alpha(f)$.

Demonstração. Observe primeiro que se $g \sim f-\alpha(f)$ e $g \leq 0$, então $g$ é forma normal para $f$ e $\sup g=0$, ou seja, $g^{-1}(0) \supset \operatorname{supp}(\mu)$ para alguma $\mu T$-invariante.

Para isso, como $\mathbb{R} / \mathbb{Z}$ é compacto, basta considerar $\bar{\mu}$ uma medida maximizante e por 3.2 .3

$$
0=\int_{X} f-\alpha(f) d \bar{\mu}=\int_{X} g d \bar{\mu} .
$$

Se $g \leq 0$, então $g(x)=0$ em $\operatorname{supp}(\bar{\mu})$, como o que queríamos.

Assim, voltando a prova do corolário, suponha que existe $g$ função não-positiva cohomóloga a $f-\alpha(f)$. Pela observação acima,

$$
\operatorname{supp}(\mu) \subset g^{-1}(\sup g)=g^{-1}(0)
$$

\footnotetext{
${ }^{1} \mathrm{Um}$ conjunto de um espaço topológico é dito genérico se puder ser escrito como a interseção enumerável de conjuntos abertos densos.
} 
para alguma medida $\mu \in \mathcal{M}_{T}(\mathbb{R} / \mathbb{Z})$.

Pelo teorema anterior e pelo lema 3.2 .9 , segue que $\mathbb{R} / \mathbb{Z} \subset g^{-1}(0)$, ou seja, $g$ é identicamente nula.

Logo, para $m \in \mathcal{M}_{T}(\mathbb{R} / \mathbb{Z})$ qualquer,

$$
\begin{aligned}
\int_{X} f-\alpha(f) d m & =\int_{X} g d m=0 \\
\Rightarrow \int_{X} f d m & =\alpha(f),
\end{aligned}
$$

contrariando portanto a propriedade de genericidade da função $f$.

Com respeito a possibilidade de conseguir regularidade na função normal, vejamos o seguinte resultado:

Teorema 3.2.12. Seja $T: \mathbb{R} / \mathbb{Z} \rightarrow \mathbb{R} / \mathbb{Z}$ definida por $T(x)=2 x(\bmod 1)$. O polinômio trigonométrico $f: \mathbb{R} / \mathbb{Z} \rightarrow \mathbb{R}$ definido por

$$
\begin{aligned}
f(x) & =\cos 2 \pi x+C \operatorname{sen} 2 \pi x-1 \\
& =\sqrt{1+C^{2}} \cos 2 \pi(x-\omega)-1,
\end{aligned}
$$

onde

$$
\begin{aligned}
C & =\frac{\sum_{n=0}^{\infty} 1-\cos \left(\pi / 2^{n}\right)}{\sum_{n=0}^{\infty} \operatorname{sen}\left(\pi / 2^{n}\right)}=1.368231157 \ldots \\
\omega & =\frac{\arctan C}{2 \pi}=0.149550073,
\end{aligned}
$$

satisfaz $\max _{\mu \in M_{f}} \int_{X} f d \mu=0$ e não é cohomólogo a uma função não-negativa em $\mathcal{C}^{1}(X)$. A demonstração pode ser encontrada em [3].

No entanto, a partir de pontos fixos de um certo operador não linear $M_{f}$ introduzido a seguir, poderão ser obtidas formas normais para $f$. 
Definição 3.2.13. Sejam $T: X \rightarrow X$ aplicação sobrejetora num conjunto $X$ não vazio e $f: X \rightarrow \mathbb{R}$ alguma função. Dada função $\varphi: X \rightarrow \mathbb{R}$, então, para cada $x \in X$, defina $M_{f} \varphi(x) \in(-\infty, \infty]$ por:

$$
M_{f} \varphi(x):=\sup _{y \in T^{-1}(x)}(f+\varphi)(y) .
$$

As iteradas de $M_{f}$ podem ser expressas por:

$$
M_{f}^{n} \varphi(x):=\sup _{y \in T^{-n}(x)}\left(S_{n} f+\varphi\right)(y), \forall x \in X
$$

De fato, para quaisquer $\bar{y}=T^{-1}(x)$ e $\bar{z}=T^{-1}(\bar{y})=T^{-2}(x)$,

$$
\begin{aligned}
M_{f}^{2} \varphi(x) & =M_{f}\left(M_{f} \varphi(x)\right) \\
& =\sup _{y \in T^{-1}(x)}\left(f+M_{f} \varphi\right)(y) \geq f(\bar{y})+M_{f} \varphi(\bar{y}) \\
& =f(\bar{y})+\sup _{z \in T^{-1}(y)}(f+\varphi)(z) \geq f(\bar{y})+f(\bar{z})+\varphi(\bar{z}) \\
& =f \circ T(\bar{z})+f(\bar{z})+\varphi(\bar{z})=\left(S_{2} f+\varphi\right)(\bar{z})
\end{aligned}
$$

Além disso, pela definição de supremo, dado $\epsilon>0$, existe $y \in T^{-1}(x)$ e $z \in T^{-2}(x)$ tais que

$$
M_{f}^{2} \varphi(x)-2 \epsilon \leq f \circ T(z)+f(z)+\varphi(z)=\left(S_{2} f+\varphi\right)(z)
$$

resultando assim que $M_{f}^{2} \varphi(x):=\sup _{y \in T^{-2}(x)}\left(S_{2} f+\varphi\right)(y), \forall x \in X$.

Com um argumento análogo, a prova para $n$ qualquer é feita por indução.

Lema 3.2.14. Sejam $T: X \rightarrow X$ aplicação sobrejetora num conjunto $X$ não vazio $e$ $f: X \rightarrow \mathbb{R}$ alguma função. Se existem $c \in \mathbb{R}$ e uma função limitada $\varphi: X \rightarrow \mathbb{R}$ tais que

$$
M_{f} \varphi=\varphi+c
$$

então $c=\lim _{n \rightarrow \infty} \frac{1}{n} \sup _{x \in X} S_{n} f(x)=\delta(f)$. 
Demonstração. Primeiramente, para todo $x \in X$ :

$$
\begin{aligned}
\varphi(x)=M_{f} \varphi(x)-c & =\left(\sup _{y \in T^{-1}(x)}(f+\varphi)(y)\right)-c \\
& =\sup _{y \in T^{-1}(x)}((f-c)+\varphi)(y)=M_{f-c} \varphi(x) .
\end{aligned}
$$

e assim, a equação $M_{f} \varphi=\varphi+c$ é equivalente a $M_{f-c} \varphi=\varphi$. Consequentemente, $\varphi=M_{f-c}^{n} \varphi$, ou seja,

$$
\varphi=-n c+\sup _{y \in T^{-n}(x)}\left(S_{n} f+\varphi\right)(y)
$$

para todo $n \in \mathbb{N}, x \in X$.

Por hipótese, $\varphi$ é limitada, e escrevemos $a \leq \varphi \leq b$ onde $a=\inf \varphi$ e $b=\sup \varphi$. Logo,

$$
\begin{gathered}
a \leq-n c+\sup _{y \in T^{-n}(x)}\left(S_{n} f+\varphi\right)(y) \leq b \\
\frac{a}{n}+c \leq \frac{1}{n} \sup _{y \in T^{-n}(x)}\left(S_{n} f+\varphi\right)(y) \leq \frac{b}{n}+c,
\end{gathered}
$$

para todo $n \in \mathbb{N}, x \in X$.

Usando o inf $\varphi$ e a segunda parte da desigualdade anterior:

$$
\begin{aligned}
& \frac{a}{n}+\frac{1}{n} \sup _{y \in T^{-n}(x)} S_{n} f(y) \leq \frac{1}{n} \sup _{y \in T^{-n}(x)}\left(S_{n} f+\varphi\right)(y) \leq \frac{b}{n}+c \\
\Rightarrow & \frac{1}{n} \sup _{y \in T^{-n}(x)} S_{n} f(y) \leq \frac{b-a}{n}+c, \text { para todo } n>0 \text { e } x \in X .
\end{aligned}
$$

Analogamente,

$$
\frac{1}{n} \sup _{y \in T^{-n}(x)} S_{n} f(y) \geq \frac{a-b}{n}+c, \text { para todo } n>0 \text { e } x \in X .
$$

Em particular,

$$
\begin{gathered}
\frac{a-b}{n}+c \leq \frac{1}{n} \sup _{x \in X} \sup _{y \in T^{-n}(x)} S_{n} f(y) \leq \frac{b-a}{n}+c \\
\Rightarrow \frac{a-b}{n}+c \leq \frac{1}{n} \sup _{y \in X} S_{n} f(y) \leq \frac{b-a}{n}+c
\end{gathered}
$$


para todo $n>0$. Fazendo $n \rightarrow \infty$, concluímos o que queríamos,

$$
c=\lim _{n \rightarrow \infty} \frac{1}{n} \sup _{x \in X} S_{n} f(x)=\delta(f) .
$$

Definição 3.2.15. Seja $X$ um espaço topológico. Suponha que $T: X \rightarrow X$ é uma função sobrejetora contínua, e $f: X \rightarrow \mathbb{R}$ é contínua. Se $\varphi \in \mathcal{C B}(X)$ é um ponto fixo de $M_{f-\delta(f)}$, então a função $\tilde{f}:=f+\varphi-\varphi \circ T$ é chamada uma forma do ponto fixo para $f$.

Lema 3.2.16. Seja $X$ um espaço topológico. Suponha que $T: X \rightarrow X$ é uma função sobrejetora contínua, e $f: X \rightarrow \mathbb{R}$ é contínua.

1. Se $\tilde{f}$ é uma forma do ponto fixo para $f$, então $\tilde{f} \leq \delta(f)$.

2. Se além disso, $X$ é compacto e metrizável, então qualquer forma do ponto fixo é também uma forma normal.

\section{Demonstração.}

1. Por hipótese, $\tilde{f}:=f+\varphi-\varphi \circ T$ para alguma $\varphi \in \mathcal{C B}(X)$ que satisfaz:

$$
\varphi(x)+\delta(f)=\sup _{y \in T^{-1}(x)}(f+\varphi)(y), \text { para todo } x \in X .
$$

Substituindo $x \in X$ por $T(x) \in X$, temos

$$
\varphi \circ T(x)+\delta(f)=\sup _{y \in T^{-1}(T(x))}(f+\varphi)(y), \text { para todo } x \in X .
$$

Ao adicionar $f+\varphi$, resulta

$$
\delta(f)-\underbrace{\left(\sup _{y \in T^{-1}(T(x))}(f+\varphi)(y)-(f+\varphi)(x)\right)}_{\geq 0}=(f+\varphi-\varphi \circ T)(x)=\tilde{f} .
$$

Logo, $\tilde{f} \leq \delta(f)$. 
2. Adicionalmente, $X$ é compacto. Por 2.2.1, $\alpha(f)=\beta(f)=\gamma(f)=\delta(f)$, donde, pelo item $1, \sup \tilde{f} \leq \alpha(f)$. Como $f$ e $\tilde{f}$ são cohomólogas, usando 3.2.7, $\alpha(f)=\sup \tilde{f}$.

Seja $\mu$ uma medida f-maximizante (sua existência é garantida por 2.3.1). Considere $A:=\{x \in X: \tilde{f}(x)=\sup \tilde{f}\}$.

Se $\tilde{f}$ não é uma forma normal então toda $m \in \mathcal{M}_{T} \operatorname{satisfaz} \operatorname{supp}(m) \not \subset \tilde{f}^{-1}(\sup \tilde{f})$. Em particular, isso vale para $\mu$, então $\mu(A)<1$ e $\mu\left(A^{C}\right)<1$. Logo,

$$
\begin{aligned}
\int_{X} f d \mu & =\int_{A} f d \mu+\int_{A^{C}} f d \mu \\
& <\sup \tilde{f} \cdot\left(\mu(A)+\mu\left(A^{C}\right)\right) \\
& =\sup \tilde{f}=\alpha(f),
\end{aligned}
$$

ou seja, $\mu \notin \mathcal{M}_{\max }$, o que é uma contradição.

O lema anterior implica que, quando $X$ é compacto e metrizável, para encontrar uma forma normal para $f$, é suficiente encontrar uma forma do ponto fixo.

Se $X$ não é compacto então isso não é válido, mesmo quando $\alpha(f)=\delta(f) \neq \pm \infty$, como veremos a seguir:

Exemplo 3.2.17. Seja $X=\mathbb{N}^{\mathbb{N}}$, isto é, o conjunto de todas as sequências $x=\left(x_{n}\right)_{n=1}^{\infty}$ de inteiros estritamente positivos.

Consideremos a função shift $T: X \rightarrow X$ definida por $T\left(x_{1}, x_{2}, x_{3}, \ldots\right)=\left(x_{2}, x_{3}, \ldots\right)$. Se $w=\left(w_{1}, \ldots, w_{k}\right)$, então o cilindro de comprimento $k$ correspondente ( $k$-cilindro) é o conjunto $[w]:=\left\{x \in X: x_{i}=w_{i}\right.$ para $\left.1 \leq i \leq k\right\}$.

Defina $f: X \rightarrow \mathbb{R}$ constante sobre 2-cilindros por:

$$
f[m, n]=\left\{\begin{array}{l}
-\frac{1}{n(n+1)}, \text { se } m=n+1 \\
-1, \text { se } m \neq n+1
\end{array} .\right.
$$


Seja $\varphi \in \mathcal{C B}(X)$ constante sobre 1-cilindros, definida por:

$$
\varphi[n]=-\frac{1}{n}, n \in \mathbb{N}
$$

Vamos encontrar o valor de $f+\varphi-\varphi \circ T$ que será necessário a seguir. Calculemos nos 2-cilindros da forma $[m, n]$.

- se $m=n+1$ :

$$
\begin{aligned}
(f+\varphi-\varphi \circ) T[m, n] & =f[n+1, n]+\varphi[n+1, n]-\varphi[n] \\
& =-\frac{1}{n(n+1)}-\frac{1}{n+1}+\frac{1}{n}=0 .
\end{aligned}
$$

- se $m \neq n+1$ :

$$
\begin{aligned}
(f+\varphi-\varphi \circ T)[m, n] & =f[m, n]+\varphi[m, n]-\varphi[n] \\
& =-1-\frac{1}{m}+\frac{1}{n}<0 .
\end{aligned}
$$

Primeiramente observemos que $\varphi$ é um ponto fixo para $M_{f-\delta(f)}$, pois satisfaz $M_{f} \varphi=\varphi+c \operatorname{com} c=\delta(f)=0$. De fato,

$$
\begin{aligned}
\left(M_{f} \varphi-\varphi\right)(x) & =\sup _{y \in T^{-1}(x)}(f+\varphi)(y)-\varphi(x) \\
& =\sup _{y \in T^{-1}(x)}(f(y)+\varphi(y)-\varphi(x)) \\
& =\sup _{y \in T^{-1}(x)}(f+\varphi-\varphi \circ T)(y)=0
\end{aligned}
$$

pelo feito anteriormente. Logo, f tem forma do ponto fixo.

Por 3.1.5, $\alpha(f) \leq \delta(f)=0$.

A fim de estimar $\alpha(f)$, denotemos por $v_{n}=\frac{1}{n} \sum_{j=0}^{n-1} \delta_{T^{j}\left(x^{(n)}\right)}$ a medida invariante suportada na órbita periódica gerada por $x^{(n)}=\overline{(n, n-1, \ldots, 1)}$. 
Note que

$$
\begin{aligned}
\int_{X} f d v_{n} & =\int_{X} f d\left(\frac{1}{n} \sum_{j=0}^{n-1} \delta_{T^{j}\left(x^{(n)}\right)}\right) \\
& =\frac{1}{n} \sum_{j=0}^{n-1} \int_{X} f d \delta_{T^{j}\left(x^{(n)}\right)}=\frac{1}{n} \sum_{j=0}^{n-1} f \circ T^{j}\left(x^{(n)}\right) \\
& =-\frac{1}{n}\left[\sum_{j=0}^{n-2} \frac{1}{(n-j-1)(n-j)}\right]-\frac{1}{n} \\
& =-\frac{1}{n} \underbrace{\left[\sum_{j=0}^{n-2} \frac{1}{n-j-1}-\frac{1}{n-j}\right]}_{\text {série telescópica }}-\frac{1}{n} \\
& =-\frac{1}{n}\left[-\frac{1}{n}+1\right]-\frac{1}{n}=\frac{1-2 n}{n^{2}}
\end{aligned}
$$

donde

$$
\alpha(f)=\sup _{m \in \mathcal{M}_{f}} \int_{X} f d m \geq \sup _{n \geq 1} \int_{X} f d v_{n}=0,
$$

e portanto $\alpha(f)=0=\delta(f)$.

Como $f<0$ então $\int_{X} f d m<0$ para toda $m \in \mathcal{M}_{f}$, implicando que $f$ não tem medida maximizante. Concluímos assim, por 3.2.9, que $f$ não tem forma normal.

\subsection{Funções essencialmente compactas}

Nessa seção definiremos compacidade essencial, o que garantirá que uma forma do ponto fixo é, de fato, uma forma normal.

Definição 3.3.1. Seja $T: X \rightarrow X$ uma sobrejeção contínua no espaço topológico $X$. Uma função contínua $f: X \rightarrow \mathbb{R}$ é essencialmente compacta (com respeito a $T$ ) se existem um ponto fixo $\varphi \in \mathcal{C B}(X)$ de $M_{f-\delta(f)}$ e um subconjunto $Y \subset X$ tais que 
1. $\hat{Y}:=\bigcap_{n=0}^{\infty} T^{-n} Y$ é nãa vazio e compacto;

2. $T(Y)=X$;

3. para cada $x \in X, \varphi(x)+\delta(f)=\sup _{y \in T^{-1}(x) \cap Y}(f+\varphi)(y)$.

Teorema 3.3.2. Seja $T: X \rightarrow X$ uma sobrejeção contínua num espaço polonês $X$. Se a função contínua $f: X \rightarrow \mathbb{R}$ é essencialmente compacta com $\varphi \in \mathcal{C B}(X)$ ponto fixo de $M_{f-\delta(f)}$, então $\tilde{f}=f+\varphi-\varphi \circ T$ é uma forma normal para $f$. Em particular,

$$
\mathcal{M}_{\text {max }}(f)=\left\{\mu \in \mathcal{M}_{T}: \operatorname{supp}(\mu) \subset \tilde{f}^{-1}(\sup \tilde{f})\right\} \neq \emptyset
$$

\section{Demonstração.}

É suficiente mostrar que $\tilde{f}$ é uma forma normal; a caracterização de $\mathcal{M}_{\max }(f)$ segue de 3.2.9.

Por hipótese, $f$ é essencialmente compacta, e da terceira condição, para $x \in \hat{Y}$,

$$
\varphi(x)+\delta(f)=\sup _{y \in T^{-1}(x) \cap Y}(f+\varphi)(y)=\sup _{y \in T^{-1}(x) \cap \hat{Y}}(f+\varphi)(y),
$$

pois observemos que $\hat{Y}=Y \cap T^{-1}(\hat{Y})$ pela própria definição de $\hat{Y}$.

Consequentemente, $\varphi \in \mathcal{C B}(X)$ é ponto fixo de $M_{\left.f\right|_{\hat{Y}}-\delta(f)}$, ou seja, $\left.\tilde{f}\right|_{\hat{Y}}$ é uma forma do ponto fixo para $\left.f\right|_{\hat{Y}}\left(\right.$ com respeito a $\left.T\right|_{\hat{Y}}$ ). Pela igualdade anterior e por 3.2.14,

$$
\delta\left(\left.f\right|_{\hat{Y}}\right)=\delta(f)
$$

Continuando, pelo item 1 de $3.1,\left.\sup \tilde{f}\right|_{\hat{Y}} \leq \delta\left(\left.f\right|_{\hat{Y}}\right)$. Adicionalmente,

$$
\alpha\left(\left.f\right|_{\hat{Y}}\right)=\left.\sup _{\left.m \in \mathcal{M}_{f}\right|_{\hat{Y}}} \int_{X} f\right|_{\hat{Y}} d m=\left.\sup _{\left.m \in \mathcal{M}_{f}\right|_{\hat{Y}}} \int_{X} \tilde{f}\right|_{\hat{Y}} d m \leq\left.\sup \tilde{f}\right|_{\hat{Y}},
$$

ou seja, como $\hat{Y}$ é compacto, $\delta\left(\left.f\right|_{\hat{Y}}\right)=\alpha\left(\left.f\right|_{\hat{Y}}\right) \leq\left.\sup \tilde{f}\right|_{\hat{Y}}$. Resultando assim que,

$$
\left.\sup \tilde{f}\right|_{\hat{Y}}=\delta\left(\left.f\right|_{\hat{Y}}\right)
$$


Também observemos que $\tilde{f}$ é uma forma do ponto fixo para $f$, e pelo item 1 de 3.1,

$$
\tilde{f} \leq \delta(f)
$$

Usando 3.1, 3.2, 3.3,

$$
\begin{gathered}
\tilde{f} \leq \delta(f)=\delta\left(\left.f\right|_{\hat{Y}}\right)=\left.\sup \tilde{f}\right|_{\hat{Y}} \\
\Rightarrow \sup \tilde{f} \leq\left.\sup \tilde{f}\right|_{\hat{Y}}
\end{gathered}
$$

e como a desigualdade contrária é sempre válida,

$$
\sup \tilde{f}=\left.\sup \tilde{f}\right|_{\hat{Y}}
$$

Finalmente, como $\hat{Y}$ é compacto, pelo item 2 de $3.1,\left.\tilde{f}\right|_{\hat{Y}}$ é uma forma normal para $\left.f\right|_{\hat{Y}}$. E usando a igualdade anterior,

$$
\left.\operatorname{supp}(\mu) \subset \tilde{f}\right|_{\hat{Y}} ^{-1}\left(\left.\sup \tilde{f}\right|_{\hat{Y}}\right)=\left.\tilde{f}\right|_{\hat{Y}} ^{-1}(\sup \tilde{f}) \subset \tilde{f}^{-1}(\sup \tilde{f})
$$

para alguma medida $\left.\mu T\right|_{\hat{Y}}$-invariante.

Para $\mu$ ser T-invariante, basta defini-la em $X$ como sendo nula no complementar de $\hat{Y}$. Logo, $\tilde{f}$ é uma forma normal para $f$.

Compacidade essencial é uma noção abstrata, então para sistemas específicos é útil substituir isso por condições mais fáceis de se verificar. Antes, lembraremos de alguns resultados e dos teoremas de [12] e [5] que serão úteis na última prova:

Definição 3.3.3. Sejam $X$ e $Y$ dois espaços normados ${ }^{2}$. Dizemos que $X$ e $Y$ são isométricos se existe $T:\left(X,\|\|_{X}\right) \rightarrow\left(Y,\|\|_{Y}\right)$ bijeção tal que $\|T(x)\|_{Y}=\|x\|_{X}$.

Teorema 3.3.4. (ponto fixo de Banach) Seja $(X, d)$ um espaço métrico, onde $X \neq \emptyset$. Suponha que $X$ é completo e seja $f: X \rightarrow X$ uma contração em $X$, isto é, existe $\lambda$ com $0 \leq \lambda<1$ tal que $d(f(x), f(y)) \leq \lambda d(x, y) \forall x, y \in X$. Então existe um único ponto fixo $p \in X$ para $f$.

\footnotetext{
${ }^{2}$ Lembremos que $\left(X,\|\|_{X}\right)$ denota o par: espaço $X$ com norma \|\|$_{X}$.
} 
Definição 3.3.5. Seja $X$ um espaço topológico. Dizemos que $H \subset X$ é relativamente compacto se $\bar{H}$ é compacto. Lembramos que $\bar{H}$ é o fecho de $H$.

Definição 3.3.6. Uma família $H$ de funções definidas em $X$ é equicontínua no ponto $x_{0} \in X$ se, dado $\epsilon>0$, existe $\delta>0$ tal que, se $d\left(x, x_{0}\right)<\delta$, então para qualquer $f \in H$,

$$
d\left(f(x), f\left(x_{0}\right)\right)<\epsilon .
$$

Teorema 3.3.7. (Arzelà-Ascoli) Seja $X$ um espaço métrico. Dizemos que $H \subset \mathcal{C}(X, \mathbb{R})$, ou seja, uma família de funções contínuas definidas em $X$ com valores em $\mathbb{R}$, é relativamente compacto se, e somente se:

1. para todo $x \in X$, o conjunto $H(x)$ é um conjunto limitado;

2. H é equicontínua.

Definição 3.3.8. Seja $X=\mathbb{N}^{\mathbb{N}}$ e $g: X \rightarrow \mathbb{R}$. Para $x, y \in X$, definimos:

$$
\begin{aligned}
& \operatorname{var}_{0}(g)=\sup g-\inf g \\
& \operatorname{var}_{1}(g)=\sup _{x_{1}=y_{1}}|g(x)-g(y)| .
\end{aligned}
$$

Se $X=\mathbb{N}^{\mathbb{N}}$, para $x \in X$ e um inteiro $n \geq 1$, o elemento $y \in X$ com $y_{1}=n$ e $y_{i}=x_{i-1}$ para todo $i \geq 2$ será denotado por $n x$.

Lema 3.3.9. $\mathbb{N}^{\mathbb{N}}$ é separável e completo com respeito a métrica $d(x, y)=2^{-\min \left\{n: x_{n} \neq y_{n}\right\}}$.

\section{Demonstração.}

Mostremos que $X=\mathbb{N}^{\mathbb{N}}$ é separável:

Consideremos $A=\{x \in X$ tal que $x$ é periódica $\}$. A é claramente enumerável.

Dado $\epsilon>0$, existe $i \in \mathbb{N}$ tal que $2^{-i}<\epsilon$. Se $x=\left(x_{1}, x_{2}, \ldots\right) \in X$ e considere $a \in A$ a sequência periódica gerada por $\overline{\left(x_{1}, \ldots, x_{i-1}\right)}$, então:

$$
d(x, a)=2^{-i}<\epsilon, \text { onde } i=\min \left\{n, x_{n} \neq a_{n}\right\},
$$


implicando que $A$ é um subconjunto enumerável e denso em $X$.

Mostremos que $X=\mathbb{N}^{\mathbb{N}}$ é completo:

Seja $\left(x_{n}\right) \subset X$ sequência de Cauchy qualquer, com $x_{n}=\left(\xi_{1}^{(n)}, \xi_{2}^{(n)}, \ldots, \xi_{k}^{(n)}, \ldots\right)$, $k, n \in \mathbb{N}$.

Dado $\epsilon>0$, existe $N_{0} \in \mathbb{N}$ tal que se $n, m>N_{0}, d\left(x_{n}, x_{m}\right)=2^{-i}<\epsilon$, onde $i=\min \left\{j, \xi_{j}^{(n)} \neq \xi_{j}^{(m)}\right\}$, ou seja, $\xi_{k}^{(n)}=\xi_{k}^{(m)}$ para $k<i$.

Para qualquer $k$ fixo, $\left\{\xi_{k}^{(n)}\right\}_{n \in \mathbb{N}}$ é de Cauchy em $\mathbb{N}$. Basta tomar $n, m>N_{0}$ e $\epsilon=2^{-k}$ para que $\left|\xi_{k}^{(n)}-\xi_{k}^{(m)}\right|=0$, e como $\mathbb{N}$ é completo, $\lim _{n \rightarrow \infty} \xi_{k}^{(n)}=\bar{x}_{k} \in \mathbb{N}$. Logo, $\bar{x}=\left(\bar{x}_{1}, \bar{x}_{2}, \ldots\right) \in X$. Mais ainda, $\bar{x}_{k}=\xi_{k}^{(n)}=\xi_{k}^{(m)}$ para $k<i$ e $m, n>N_{0}$.

Consequentemente, tomando $n>N_{0}$,

$$
d\left(x_{n}, \bar{x}\right)=2^{-\min \left\{j, \xi_{j}^{(n)} \neq \bar{x}_{j}\right\}}=2^{-i}<\epsilon,
$$

ou seja, $x_{n} \rightarrow \bar{x} \in X$.

Finalmente, o teorema de Jenkinson, Mauldin e Urbański, encontrados em [6], enuncia o que queríamos.

Teorema 3.3.10. Seja $X=\mathbb{N}^{\mathbb{N}}$ e $T: X \rightarrow X$ a aplicação shift. Suponha que $f: X \rightarrow$ $\mathbb{R}$ é limitada superiormente e constante nos 2-cilindros, e que existe $n \geq 1$ tal que

$$
\operatorname{var}_{1}(f)<\left.\inf f\right|_{[n]}-\left.\sup f\right|_{[i]}
$$

para todo $i \geq 1$ suficientemente grande.

Então $f$ é essencialmente compacta, portanto tem uma forma normal $\tilde{f}, e$

$$
\mathcal{M}_{\text {max }}(f)=\left\{\mu \in \mathcal{M}_{T}: \operatorname{supp}(\mu) \subset \tilde{f}^{-1}(\sup \tilde{f})\right\} \neq \emptyset
$$

\section{Demonstração.}

Considere o espaço $K(X)$ das funções $\varphi: X \rightarrow \mathbb{R}$ limitadas que são constantes nos 1-cilindros. A seguir, faremos a identificação do espaço das funções limitadas que 
são constantes nos 1-cilindros com o espaço das sequências $\ell^{\infty}$, equipado com a norma usual.

Seja $F: K \rightarrow l^{\infty}$ dada por $F(\varphi)=x=\left(x_{1}, x_{2}, \ldots, x_{n}, \ldots\right)$, onde $x_{i}=\varphi[i]$, para $i \geq 1$.

$F$ está bem definida e é bijetora. De fato,

$$
\|F \varphi\|_{\infty}=\|x\|_{\infty}=\sup _{m \in \mathbb{N}}\left|x_{m}\right|=\sup _{m \in \mathbb{N}}|\varphi[m]|=\sup _{x \in X}|\varphi(x)|<\infty .
$$

implicando que $F(\varphi) \in l^{\infty}$ e, por 3.3.3, temos também que os espaços são isométricos.

Inicialmente, para utilizar (3.3.4) em $M_{f}$ verifiquemos que:

- A função $M_{f}$ preserva o espaço $K$.

Considere $\varphi \in K$. Como f é constante nos 2-cilindros,

$$
\begin{aligned}
M_{f}(\varphi(x)) & =\sup _{y \in T^{-1}(x)}(f+\varphi)(y) \\
& =\sup _{m \in \mathbb{N}}(f(m x)+\varphi(m x))
\end{aligned}
$$

depende só da primeira coordenada de $x$, ou seja, $M_{f}(\varphi)$ é constante nos 1-cilindros.

Além disso, como $f$ é limitada superiormente,

$$
M_{f}(\varphi(x)) \leq \sup _{m \in \mathbb{N}} f(m x)+\sup _{m \in \mathbb{N}} \varphi(m x)<\infty
$$

e por 3.4, para $i$ suficientemente grande,

$$
\begin{aligned}
M_{f}(\varphi(x)) & \geq\left.\inf (f+\varphi)\right|_{[n]} \\
& \geq\left.\inf f\right|_{[n]}+\inf \varphi \\
& \geq \operatorname{var}_{1}(f)+\left.\sup f\right|_{[i]}+\inf \varphi \geq-\infty
\end{aligned}
$$

ou seja, $M_{f}(\varphi)$ é limitada.

- O espaço é completo, já que $l^{\infty}$ é um espaço de Banach. 
- Para $0 \leq \lambda<1$, a função $\varphi \mapsto M_{f, \lambda}(\varphi)=M_{f}(\lambda \varphi)$ é $\lambda$-Lipschitz em $l^{\infty}$.

$$
\begin{aligned}
\left\|M_{f, \lambda} \varphi_{1}-M_{f, \lambda} \varphi_{2}\right\|_{\infty} & =\sup _{x \in X}\left|\sup _{y \in T^{-1}(x)}\left(f+\lambda \varphi_{1}\right)(y)-\sup _{y \in T^{-1}(x)}\left(f+\lambda \varphi_{2}\right)(y)\right| \\
& \leq \sup _{x \in X}\left|\sup _{m \in \mathbb{N}}\left(f+\lambda \varphi_{1}-f-\lambda \varphi_{2}\right)(m x)\right| \\
& =\sup _{x \in X}\left|\sup _{m \in \mathbb{N}} \lambda\left(\varphi_{1}-\varphi_{2}\right)(m x)\right| \\
& =\lambda \sup _{x \in X}\left|\sup _{m \in \mathbb{N}}\left(\varphi_{1}-\varphi_{2}\right)(m x)\right| \\
& =\lambda \sup _{x \in X}\left|\left(\varphi_{1}-\varphi_{2}\right)(x)\right| \\
& =\lambda\left\|\varphi_{1}-\varphi_{2}\right\|_{\infty} .
\end{aligned}
$$

Portanto, para $0 \leq \lambda<1, M_{f, \lambda}$ tem um único ponto fixo $\varphi_{\lambda}$, ou seja,

$$
\begin{gathered}
M_{f, \lambda}\left(\varphi_{\lambda}\right)=\varphi_{\lambda} \\
\Rightarrow \sup _{y \in T^{-1}(x)}\left(f(y)+\lambda \varphi_{\lambda}(y)\right)=\sup _{m \in \mathbb{N}}\left(f(m x)+\lambda \varphi_{\lambda}(m x)\right)=\varphi_{\lambda}(x) .
\end{gathered}
$$

Além disso,

$$
\operatorname{var}_{0}\left(\varphi_{\lambda}\right) \leq \operatorname{var}_{1}(f)
$$

para todo $0 \leq \lambda<1$, pois

$$
\begin{aligned}
\varphi_{\lambda}(x)-\varphi_{\lambda}(y) & =\sup _{m \in \mathbb{N}}\left(f(m x)+\lambda \varphi_{\lambda}(m x)\right)-\sup _{m \in \mathbb{N}}\left(f(m y)+\lambda \varphi_{\lambda}(m y)\right) \\
& \leq \sup _{m \in \mathbb{N}}\left(f(m x)+\lambda \varphi_{\lambda}(m x)-f(m y)+\lambda \varphi_{\lambda}(m y)\right) \\
& =\sup _{m \in \mathbb{N}}(f(m x)+\lambda \underbrace{\left(\varphi_{\lambda}(m x)-\varphi_{\lambda}(m y)\right)}_{\varphi \text { é constante nos 1-cilindros }}-f(m y)) \\
& =\sup _{m \in \mathbb{N}}(f(m x)-f(m y))=\operatorname{var}_{1}(f) \\
\Rightarrow & \sup _{x, y \in X}\left(\varphi_{\lambda}(x)-\varphi_{\lambda}(y)\right) \leq \operatorname{var}_{1}(f) \\
\Rightarrow & \operatorname{var}_{0}\left(\varphi_{\lambda}\right)=\sup _{x \in X} \varphi_{\lambda}(x)-\inf _{y \in Y} \varphi_{\lambda}(y) \leq \operatorname{var}_{1}(f) .
\end{aligned}
$$


Consideremos agora, para $0 \leq \lambda<1, \varphi_{\lambda}^{*}:=\varphi_{\lambda}-\inf \varphi_{\lambda}$.

$$
\begin{aligned}
\left\|\varphi_{\lambda}^{*}\right\| & =\sup _{x \in X}\left|\varphi_{\lambda}^{*}(x)\right|=\sup _{x \in X}\left|\left(\varphi_{\lambda}-\inf \varphi_{\lambda}\right)(x)\right| \\
& =\sup _{x \in X}\left(\varphi_{\lambda}-\inf \varphi_{\lambda}\right)(x)=\sup \varphi_{\lambda}-\inf \varphi_{\lambda} \\
& =\operatorname{var}_{0}\left(\varphi_{\lambda}\right) \stackrel{3.5}{\leq} \operatorname{var}_{1}(f) .
\end{aligned}
$$

Além disso, como $\varphi_{\lambda}^{*}$ são constantes nos 1-cilindros, claramente $\left(\varphi_{\lambda}^{*}\right)_{0 \leq \lambda<1}$ é equicontínua. Logo, utilizando 3.3.7 temos que $\left(\varphi_{\lambda}^{*}\right)_{0 \leq \lambda<1}$ é relativamente compacto em $\ell^{\infty}$. Lembremos que, como o espaço é completo, isso é equivalente a toda sequência de pontos de $X$ conter uma subsequência convergente em $\ell^{\infty}$ [5]. Segue que, existe sequência $\lambda_{i}$ tal que

$$
\lim _{\lambda_{i} \rightarrow 1} \varphi_{\lambda_{i}}^{*}=\bar{\varphi}
$$

e,

$$
\lim _{\lambda_{i} \rightarrow 1} M_{f, \lambda_{i}} \varphi_{\lambda_{i}}^{*}=M_{f} \bar{\varphi} .
$$

Também temos que, se $0 \leq \lambda<1$,

$$
\begin{aligned}
M_{f, \lambda} \varphi_{\lambda}^{*}(x) & =M_{f, \lambda}\left(\varphi_{\lambda}-\inf \varphi_{\lambda}\right)(x) \\
& =\sup _{y \in T^{-1}(x)}\left(f+\lambda\left(\varphi_{\lambda}-\inf \varphi_{\lambda}\right)\right)(y) \\
& =\sup _{y \in T^{-1}(x)}\left(f+\lambda \varphi_{\lambda}\right)(y)-\lambda \inf \varphi_{\lambda}+\inf \varphi_{\lambda}-\inf \varphi_{\lambda} \\
& =M_{f, \lambda} \varphi_{\lambda}(x)-\inf \varphi_{\lambda}+(1-\lambda) \inf \varphi_{\lambda} \\
& =\left(\varphi_{\lambda}-\inf \varphi_{\lambda}\right)+(1-\lambda) \inf \varphi_{\lambda} \\
& =\varphi_{\lambda}^{*}(x)+(1-\lambda) \inf \varphi_{\lambda}
\end{aligned}
$$

para todo $x \in X$. Logo,

$$
\lim _{\lambda_{i} \rightarrow 1}\left(\left(1-\lambda_{i}\right) \inf \varphi_{\lambda_{i}}\right)=0
$$

e,

$$
M_{f} \bar{\varphi}=\bar{\varphi}+\delta(f) .
$$


Note que, analogamente a 3.5,

$$
\operatorname{var}_{0}(\bar{\varphi}) \leq \operatorname{var}_{1}(f)
$$

Portanto, para $x \in X$ e $i>I_{0}$,

$$
\begin{gathered}
\bar{\varphi}(i x)-\bar{\varphi}(n x) \leq \operatorname{var}_{0}(\bar{\varphi}) \leq \operatorname{var}_{1}(f)<\left.\inf f\right|_{[n]}-\left.\sup f\right|_{[i]} \leq f(n x)-f(i x) \\
\Rightarrow(f+\bar{\varphi})(i x)<(f+\bar{\varphi})(n x) \text { para todo } x \in X \text { e } i>I_{0}>n .
\end{gathered}
$$

Em outros termos, se $J>n$ satisfaz $\operatorname{var}_{1}(f)<\left.\inf f\right|_{[n]}-\left.\sup f\right|_{[i]}$ para $i>J$, então

$$
M_{f} \bar{\varphi}(x)=\sup _{y \in T^{-1}(x)}(f+\bar{\varphi})(y)=\max _{1 \leq j \leq J}(f+\bar{\varphi})(j x)
$$

para todo $x \in X$.

Notemos que $f$ é essencialmente compacta. Por 3.6, existe $\bar{\varphi} \in \mathcal{C B}(X)$ ponto fixo de $M_{f-\delta(f)}$. Ao considerar $Y=\bigcup_{j=1}^{J}[j]$,

1. Para $x \in X$,

$$
\begin{aligned}
\hat{Y}: & =\bigcap_{n=0}^{\infty} T^{-n} Y \\
& =\left(\bigcup_{j=1}^{J}[j]\right) \bigcap\left(\bigcup_{j=1, \ldots, J}\left[x_{1}, j\right]\right) \bigcap\left(\bigcup_{j=1, \ldots, J}\left[x_{1}, x_{2}, j\right]\right) \bigcap \ldots \\
& =\bigcup_{j_{i}=1}^{J}\left[j_{1}, j_{2}, j_{3}, \ldots\right]=\{1,2, \ldots, J\}^{\mathbb{N}}
\end{aligned}
$$

é não vazio e compacto.

2. $T(Y)=X$.

3. para cada $x \in X$,

$$
M_{f} \bar{\varphi}(x) \stackrel{3.6}{=} \bar{\varphi}+\delta(f) \stackrel{3.7}{=} \max _{1 \leq j \leq J}(f+\bar{\varphi})(j x)=\sup _{y \in T^{-1}(x) \cap Y}(f+\bar{\varphi})(y) .
$$


Finalmente, como a aplicação shift em $X$ é sobrejetora e contínua (com respeito a métrica definida) e $X=\mathbb{N}^{\mathbb{N}}$ é separável e completo (portanto é um espaço polonês), ao utilizar 3.3.2 concluimos que $\tilde{f}$ é uma forma normal para $f$.

Exemplo 3.3.11. Considere $X=\mathbb{N}^{\mathbb{N}}$ e $T: X \rightarrow X$ a aplicação shift. Seja $f: X \rightarrow \mathbb{R}$ constante nos 2-cilindros, definida por $f[i, j]=\left.\max g\right|_{C(i, j)}$, onde $g:[0,1] \rightarrow \mathbb{R}$ é dada por $g(x)=x(1-x) e$

$$
C(i, j)=\left[\frac{1}{i+1+\frac{1}{j}}, \frac{1}{i+\frac{1}{j+1}}\right] \subset[0,1]
$$

para $i, j \geq 1$.

Escolhendo $n=2$, veremos que,

$$
\operatorname{var}_{1}(f)<\left.\inf f\right|_{[n]}-\left.\sup f\right|_{[i]} \text {. }
$$

Resolução 3.3.12. Analisemos $\left.f\right|_{[2]}$ :

$$
\left.f\right|_{[2]}=\left.\max g\right|_{C(2, j)}=\left.\max g\right|_{\left[\frac{1}{3+\frac{1}{j}}, \frac{1}{2+\frac{1}{j+1}}\right]}
$$

Se $j=1,\left[\frac{1}{3+\frac{1}{j}}, \frac{1}{2+\frac{1}{j+1}}\right]=\left[\frac{1}{4}, \frac{2}{5}\right]$.

Se $j \rightarrow \infty,\left[\frac{1}{3+\frac{1}{j}}, \frac{1}{2+\frac{1}{j+1}}\right] \rightarrow\left[\frac{1}{3}, \frac{1}{2}\right]$.

Implicando assim que,

$$
\left.\inf f\right|_{[2]}=\left.\max g\right|_{\left[\frac{1}{4}, \frac{2}{5}\right]}=g\left(\frac{2}{5}\right)=\frac{6}{25} .
$$

Analisemos $\left.\sup f\right|_{[i]}$ para $i$ suficientemente grande:

$$
\left.\sup f\right|_{[i]}=\left.g\left(\frac{1}{i}\right) \Rightarrow \limsup _{i \rightarrow \infty} f\right|_{[i]}=g(0)=0
$$


Logo, quando $i \rightarrow \infty$

$$
\left.\inf f\right|_{[2]}-\left.\sup f\right|_{[i]} \rightarrow \frac{6}{25}-0=\frac{6}{25}
$$

Finalizando, analisemos $\operatorname{var}_{1}(f)=\sup \left|f\left[l, j_{1}\right]-f\left[l, j_{2}\right]\right|$.

Tomando $l=1$, estaremos olhando para os intervalos que incluem o máximo $e$ mínimo da função $g$ :

$$
f[1, j]=\left.\max g\right|_{\left[\frac{1}{2+\frac{1}{j}}, \frac{1}{1+\frac{1}{j+1}}\right]} \cdot
$$

Observemos que $\frac{1}{2+\frac{1}{j}} \leq \frac{1}{2} e \frac{1}{2} \leq \frac{1}{1+\frac{1}{j+1}} \leq 1$. Então, o máximo da função $g$ (quando g assume $1 / 2$ ) está em todo os intervalos. Ou seja, $f[1, j]=g\left(\frac{1}{2}\right), \forall j \in \mathbb{N}$. Implicando assim,

$$
\begin{aligned}
\operatorname{var}_{1}(f) & =\sup \left|f\left[l, j_{1}\right]-f\left[l, j_{2}\right]\right|=\sup _{j_{1}, j_{2} \in \mathbb{N}} f\left[1, j_{1}\right]-f\left[1, j_{2}\right] \\
& =g\left(\frac{1}{2}\right)-g\left(\frac{1}{2}\right)=0 .
\end{aligned}
$$

Assim, vale 3.4. Pelo teorema anterior, $f$ é essencialmente compacta, portanto tem uma forma normal que garante a existência de uma medida f-maximizante caracterizada pelo seu suporte.

Observação 3.3.13. A função $f$ do exemplo 3.2.17 não satisfaz 3.4, pois, para $m, n \in \mathbb{N}$,

$$
-\frac{1}{2} \leq f[n+1, n]<0 \text { e } f[m, n]=-1 \text { para } m \neq n+1,
$$

resultando que:

$\left.\inf f\right|_{[n]}=-1$ para todo $n$,

$\left.\sup f\right|_{[i]}=0$ para $i$ suficientemente grande.

Como $\operatorname{var}_{1}(f)>0$, temos 


$$
\operatorname{var}_{1}(f)>\left.\inf f\right|_{[n]}-\left.\sup f\right|_{[i]}=-1,
$$

para $i$ suficientemente grande e $n$ qualquer.

Observação 3.3.14. O teorema 3.3.10 pode ser estendido a subshifts do tipo finito em alfabetos contáveis e funções $f$ de variação somável. Um estudo explorando esse assunto é encontrado em [7]. 

[1] Bartle, R. G., The elements of integration, New York, Wiley \& Sons (1966).

[2] Brézis, H., Análisis funcional: Teoría y aplicaciones, Madrid, Alianza (1984).

[3] Bousch, T. e Jenkinson, O., Cohomology classes of dynamically non-negative $C^{k}$ functions, Inventiones Mathematicae, 148 (2002), 207-217.

[4] Deuschel, J. D. e Stroock, D. W., Large Deviations, London, Academic Press (1989).

[5] Hon̈ig, C. S., Aplicações da topologia à análise, Rio de Janeiro, Impa (1976).

[6] Jenkinson, O., Mauldin, R. D. e Urbański, M., Ergodic optimization for noncompact dynamical systems, Dynamical Systems, 22 (2007), 379-388.

[7] Jenkinson, O., Mauldin, R. D. e Urbański, M., Ergodic optimization for countable alphabet subshifts of finite type, Ergodic Theory and Dynamical Systems, $\mathbf{2 6}$ (2006), 1791-1803.

[8] Jenkinson, O., Ergodic optimization, Discrete and Continuous Dynamical Systems, 15 (2006), 197-224.

[9] Jenkinson, O., Every ergodic measure is uniquely maximizing, Discrete and Continuous Dynamical Systems, 16 (2006), 383-392. 
[10] Kolmogorov, A. N. e Fomin, S. V., Elementos de la teoria de funciones y del analisis funcional, Moscou, Mir (1972).

[11] Krengel, U., Ergodic theorems, Berlin, Walter de Guyter (1985).

[12] Lima, E. L., Espaços métricos, Rio de Janeiro, Impa (1993).

[13] Mañé, R., Introdução à teoria ergódica, Rio de Janeiro, Impa (1983).

[14] Rudin, W., Real and complex analysis, New York, McGraw-Hill (1987).

[15] Vestrup, E. M., The theory of measures and integration, New Jersey, Wiley (2003).

[16] Walters, P., An introduction to Ergodic Theory, New York, Springer-Verlag (1982). 
